Aus der Klinik für Kardiologie und Pneumologie

(Prof. Dr. med. G. Hasenfuß)

der Medizinischen Fakultät der Universität Göttingen

\title{
Vorhersagbarkeit von klinischen Ereignissen bei Patienten mit einem implantierbaren Kardioverter-Defibrillator durch Auswertungen aus dem 24-Stunden-Langzeit-EKG
}

\author{
INAUGURAL-DISSERTATION \\ zur Erlangung des Doktorgrades \\ der Medizinischen Fakultät der \\ Georg-August-Universität zu Göttingen
}

vorgelegt von

Ansgar Wilhelm Wessels

aus Haselünne

Göttingen 2015 
Dekan:

Prof. Dr. Heyo K. Kroemer

I. Berichterstatter:

Prof. Dr. Markus Zabel

II. Berichterstatter:

Prof. Dr. Thomas Meyer

III. Berichterstatter:

Prof. Dr. Matthias Sigler

IV. Berichterstatterin:

Prof. Dr. Margarete Schön

Tag der mündlichen Prüfung: 06.07.2016 


\section{INHALTSVERZEICHNIS}

ABKÜRZUNGSVERZEICHNIS ............................................................................. 4

ABBILDUNGSVERZEICHNIS............................................................................ 7

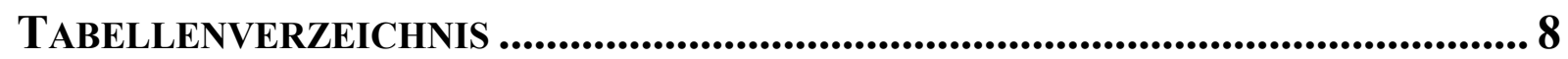

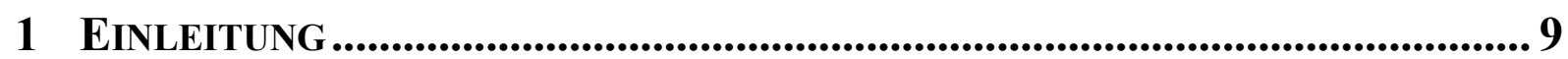

1.1 Plötzlicher Herztod und Therapie durch implantierbare Kardioverter-Defibrillatoren..... 9

1.2 Diagnostische Verfahren zur Risikostratifikation des plötzlichen Herztodes ................. 11

1.3 Risikostratifizierungsparameter im 24-h-Langzeit-Elektrokardiogramm ...................... 12

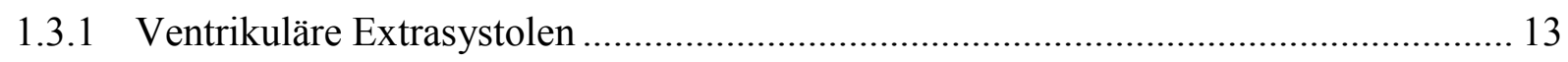

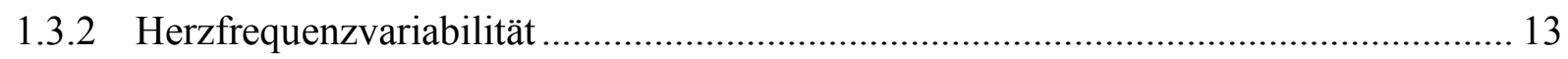

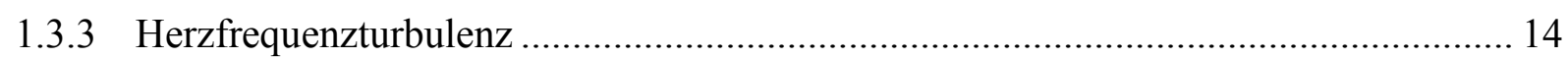

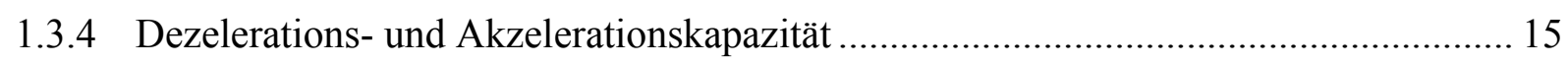

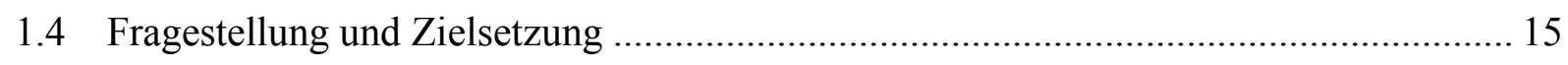

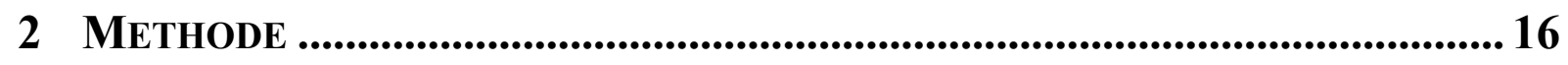

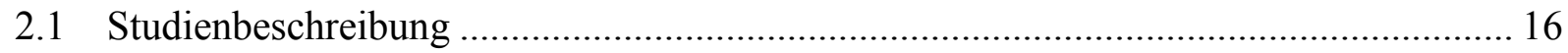

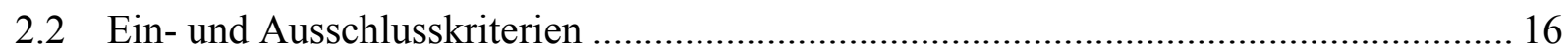



2.4 Berechnung der Herzfrequenzvariabilität .................................................................. 18

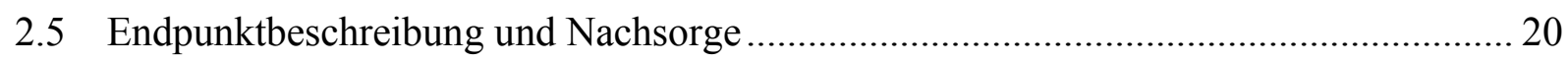

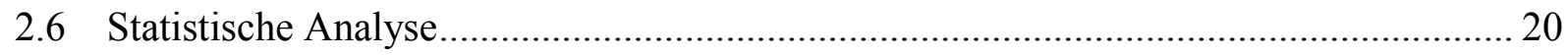

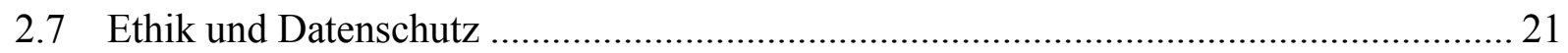

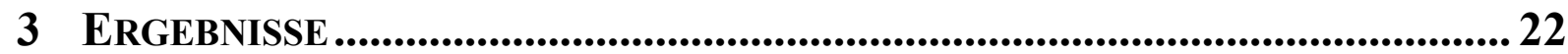

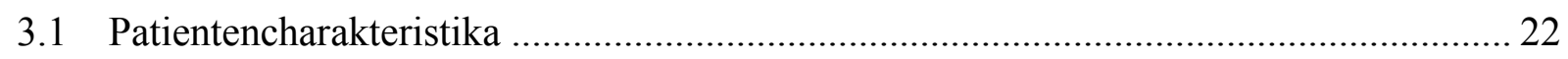

3.2 Patienten mit ICD-Neuimplantation ohne Aufnahme in das Studienregister ................ 23

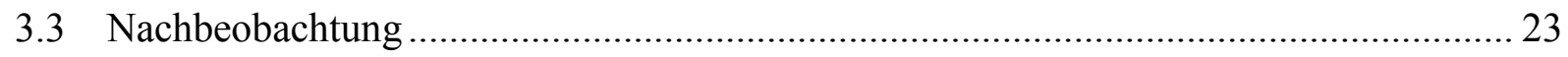

3.4 Prävalenz klinischer Ereignisse in den LZ-EKG-Risikomarkergruppen ....................... 24

3.5 Univariate Analyse zur Vorhersagbarkeit des Erreichens der Endpunkte ..................... 26

3.6 Multivariate Analyse zur Vorhersagbarkeit des Erreichens der Endpunkte .................. 28



4.1 Prognostische Relevanz von LZ-EKG-Risikomarkern für Patienten mit einem ICD..... 31

4.2 Stärken und Schwächen der vorliegenden Arbeit ..................................................... 35 


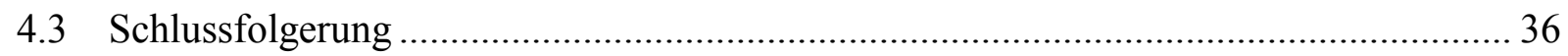

5 ZUSAMMENFASSUNG................................................................................. 37

LITERATURVERZEICHNIS....................................................................................... 38 


\section{ABKÜRZUNGSVERZEICHNIS}

AA-Intervall

Abb.

AC

ACC

AHA

DC

EKG

EPU

HF

HR

HRV

HRT

ICD

ICM

KHK

LF

LVEF

LZ-EKG

$\mathrm{ms}$
Aberrant-zu-Aberrant-Intervall

Abbildung

Akzelerationskapazität (engl. acceleration capacity)

American College of Cardiology

American Heart Association

Dezelerationskapazität (engl. deceleration capacity)

Elektrokardiogramm

elektrophysiologische Untersuchung

Band der hohen Frequenzen (engl. high frequency), Parameter der frequenzbezogenen HRV

Hazard-Ratio

Herzfrequenzvariabilität (engl. heart rate variability)

Herzfrequenzturbulenz (engl. heart rate turbulence)

implantierbarer Kardioverter-Defibrillator (engl. implantable cardioverter-defibrillator)

ischämische Kardiomyopathie (engl. ischemic cardiomyopathy)

koronare Herzkrankheit

Band der niedrigen Frequenzen (engl. low frequency), Parameter der frequenzbezogenen HRV

linksventrikuläre Ejektionsfraktion

Langzeit-Elektrokardiogramm

Millisekunde 


$\begin{array}{ll}\text { NA-Intervall } & \text { Normal-zu-Aberrant-Intervall } \\ \text { NN-Intervall } & \text { Normal-zu-Normal-Intervall } \\ \text { nsVT } & \text { nicht-anhaltende ventrikuläre Tachykardie (engl. non-sustained } \\ & \text { ventricular tachycardia) } \\ \text { NYHA } & \text { New York Heart Association } \\ \text { PRSA } & \text { phasengleichgerichtete Signalmittelung (engl. phase rectified signal } \\ & \text { averaging) } \\ \text { RMSSD } & \text { Quadratwurzel des Mittelwertes der Summe der quadrierten } \\ & \text { Differenzen aufeinanderfolgender NN-Intervalle (engl. root mean } \\ & \begin{array}{l}\text { square of successive differences between adjacent NN-intervals), } \\ \text { Parameter der zeitbezogenen HRV } \\ \text { RR }\end{array} \\ & \text { relatives Risiko } \\ & \text { R-Zacke-zu-R-Zacke-Intervall }\end{array}$

SAECG Signal-gemitteltes Elektrokardiogramm (engl. signal-averaged electrocardiogram)

SCD plötzlicher Herztod (engl. sudden cardiac death)

SD Standardabweichung (engl. standard deviation)

SDNN Standardabweichung aller NN-Intervalle (engl. standard deviation of $N N$ ), Parameter der zeitbezogenen HRV

SDNNi SDNN-Index, Parameter der zeitbezogenen HRV

SNN50+ Anzahl aller NN-Intervalle, die eine Größenänderung von über 50ms im Vergleich zum vorrausgehenden NN-Intervall aufwiesen, Parameter der zeitbezogenen HRV

Tab. Tabelle

TIA transitorische ischämische Attacke 
TO

TS

VES

VF

VLF

VT

z. B.
Turbulenzbeginn (engl. turbulence onset), Parameter der HRT

Turbulenzsteigung (engl. turbulence slope), Parameter der HRT

ventrikuläre Extrasystole

Kammerflimmern (engl. ventricular fibrillation)

Band der sehr niedrigen Frequenzen (engl. very low frequency),

Parameter der frequenzbezogenen HRV

ventrikuläre Tachykardie

zum Beispiel 


\section{ABBILDUNGSVERZEICHNIS}

Abb. 1 Ausschnitt aus dem Analysemenü des Pathfinder-Programmes 18

Abb. 2 Ereignisfreies Überleben für den kombinierten Endpunkt aus appropiatem ICDSchock und Gesamtmortalität in Abhängigkeit vom Ergebnis der nsVT-Quantifizierung $(p=0,05)$ .29 


\section{TABELLENVERZEICHNIS}

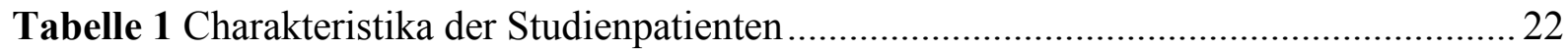

Tabelle 2 Anzahl der Patienten mit klinischen Ereignissen in den LZ-EKG-

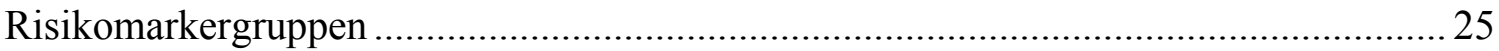

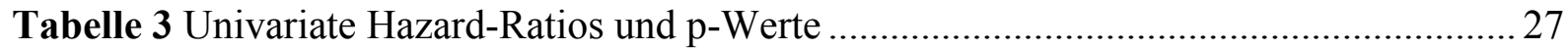

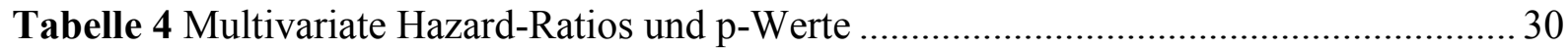




\section{Einleitung}

\subsection{Plötzlicher Herztod und Therapie durch implantierbare Kardioverter- Defibrillatoren}

Der plötzliche Herztod (SCD) ist die häufigste Todesursache in den westlichen Industrienationen (Myerburg et al. 1993; Myerburg et al. 1997). Er ist definiert als Tod kardialer Genese, welcher innerhalb einer Stunde aus völligem Wohlbefinden auftritt (Pratt et al. 1996). Die Mehrheit dieser Todesfälle ist durch anhaltende ventrikuläre Tachykardien (VT) oder Kammerflimmern (VF) mit konsekutivem Herz-Kreislauf-Stillstand bedingt (Bayès de Luna et al. 1989; Myerburg et al. 1997; Zipes und Wellens 1998).

Die strukturelle Herzerkrankung ist der Hauptrisikofaktor für den SCD. Etwa die Hälfte aller Patienten, die am SCD verstirbt, hat eine eingeschränkte linksventrikuläre Ejektionsfraktion (LVEF). Die größte Gruppe innerhalb der Patienten mit einer strukturellen Herzerkrankung wird von Patienten mit einer koronaren Herzerkrankung gebildet. Ein besonderes Risiko besteht bei Postmyokardinfarktpatienten, gefolgt von nicht-ischämischen Kardiomyopathien (Huikuri et al. 2001; Myerburg et al. 1997).

Eine Therapieoption zur Prophylaxe eines SCDs stellt der implantierbare KardioverterDefibrillator (ICD) dar. Ein ICD wird in der Regel ähnlich wie ein Herzschrittmacher auf dem Brustmuskel des Patienten eingepflanzt und ist in der Lage, durch ein transvenöses Elektrodensystem ventrikuläre Tachykardien und Kammerflimmern zu detektieren und wirksam durch Überstimulation oder Schock-Entladung zu therapieren (DiMarco 2003; Mirowski et al. 1980; Wathen et al. 2001).

Die drei wesentlichen Studien, die eine Mortalitätsreduktion durch ICD-Implantation zur Sekundärprophylaxe des SCDs aufgezeigt haben, sind die AVID-Studie (Antiarrhythmics versus Implantable Defibrillators), die CASH-Studie (The Cardiac Arrest Study Hamburg) und die CIDS-Studie (Canadian Implantable Defibrillator Study) (Connolly et al. 2000a; Connolly et al. 2000b; Kuck et al. 2000; AVID-Forscher 1997). Eine ICD-Implantation zur Sekundärprophylaxe wird nach überlebtem SCD durch ventrikuläre Tachyarrhythmien ohne vermeidbare Ursachen, dem spontanen Auftreten anhaltender ventrikulärer Tachykardien bei struktureller Herzerkrankung oder nach unklaren Synkopen bei Induzierbarkeit von hämodynamisch relevanten anhaltenden ventrikulären Tachykardien durch eine elektrophysiologische Untersuchung (EPU) in den aktuellen gemeinsamen Leitlinien des 
American College of Cardiology und der American Heart Association (ACC/AHA) empfohlen (Epstein et al. 2008).

Eine Mortalitätsreduktion durch eine primärprophylaktische ICD-Implantation für Patienten mit einer hochgradig eingeschränkten LVEF und weiteren Risikofaktoren wurde durch die MADIT-I-Studie (Multicenter Automatic Defibrillator Implantation Trial), die MADIT-IIStudie, die MUSTT-Studie (Multicenter Unsustained Tachycardia Trial), die DEFINITEStudie (The Defibrillators in Non-Ischemic Cardiomyopathy Treatment Evaluation) und die SCD-HeFT-Studie (Sudden Cardiac Death in Heart Failure Trial) aufgezeigt (Bardy et al. 2005; Buxton et al. 1999; Kadish et al. 2004; Moss et al. 1996; Moss et al. 2002). In den aktuellen ACC/AHA-Leitlinien besteht die Indikation der ICD-Implantation zur Primärprophylaxe für Patienten mit einer LVEF $\leq 35 \%$ bei ischämischer oder nichtischämischer Kardiomyopathie im NYHA-Stadium II-III sowie für Postmyokardinfarktpatienten mit einer LVEF $\leq 30 \%$ im NYHA-Stadium I und für Postmyokardinfarktpatienten mit einer LVEF $\leq 40 \%$ bei Induzierbarkeit von ventrikulären Tachyarrhythmien durch eine EPU (Epstein et al. 2008).

Im Jahr 2012 wurden circa 30.000 ICD-Implantationen in Deutschland durchgeführt (Markewitz 2014). In 2,1 \% der Fälle trat mindestens eine perioperative Komplikation auf. Die häufigsten Komplikationen waren eine Sondendislokation $(0,6 \%$ der gesamten ICDImplantationen), ein interventionspflichtiger Pneumothorax $(0,5 \%)$ ein interventionspflichtiges Taschenhämatom $(0,4 \%)$ und eine Sondendysfunktion $(0,2 \%)$ (Markewitz 2014). Die durchschnittlichen Kosten für einen ICD betragen in Deutschland $18.000 €$ (Schneider 2006).

Neben den eingriffsbedingten Risiken besteht für den ICD-Träger die Gefahr unnötiger und damit unangemessener Schockabgaben. Diese werden vor allem durch Fehlinterpretation nicht lebensbedrohender supraventrikulärer Tachykardien wie z. B. Sinustachykardien oder Vorhofflimmern ausgelöst (Daubert et al. 2008; Jodko et al. 2009). In der Literatur ist eine kumulative Fünf-Jahres-Inzidenz unangemessener Schockabgaben von $18 \%$ beschrieben (Van Rees et al. 2011). Die Abgabe eines Schocks stellt für den Patienten häufig ein belastendes Ereignis dar, das mit einer verminderten Lebensqualität und einem erhöhten Risiko der Entwicklung von Angststörungen und möglicherweise mit einer Prognoseverschlechterung assoziiert ist (Heller et al. 1998; Kamphuis et al. 2003; Poole et al. 2008; Vollmann et al. 2005). In der kürzlich veröffentlichten prospektiven MADIT-RITStudie (Multicenter Automatic Defibrillator Implantation Trial: Reduce Inappropriate 
Therapy) konnte bei 1500 ICD-Patienten nach 16 Monaten ein Rückgang dieser unangemessenen Schockabgaben und der Mortalität durch Veränderungen im Detektionsalgorithmus des ICDs aufgezeigt werden (Moss et al. 2012).

Die therapeutische Effektivität der ICD-Therapie ist, gemessen an den teilweise beträchtlichen Nebenwirkungen, relativ gering. So müssen je nach zugrundeliegender Studie 15 Patienten 20 Monate (MADIT-II-Studie), 17 Patienten 46 Monate (SCD-HeFT-Studie), 19 Patienten 29 Monate (DEFINITIE-Studie) oder 12 Patienten 18 Monate (AVID-Studie) mit einem ICD behandelt werden, um einen SCD zu verhindern (AVID-Forscher 1997; Bardy et al. 2005; Kadish et al. 2004; Moss et al. 2002).

Die genannten Nebenwirkungen und die relativ geringe therapeutische Effektivität begründen die Notwendigkeit, durch eine suffiziente SCD-Risikostratifikation Patienten mit einer aktuellen Indikation zur ICD-Implantation, jedoch niedrigem Risiko für das Auftreten lebensbedrohlicher ventrikulärer Herzrhythmusstörungen besser detektieren zu können. Ob es durch Bestimmung verschiedener Parameter aus dem 24-h-Langzeit-EKG gelingen kann, eine verbesserte Risikostratifikation für Patienten mit aktueller ICD-Indikation zu erreichen, soll im Rahmen der vorliegenden Arbeit geprüft werden.

\subsection{Diagnostische Verfahren zur Risikostratifikation des plötzlichen Herztodes}

Zur Risikostratifikation des SCD stehen verschiedene diagnostische Verfahren zur Verfügung. Ein wichtiger prognostischer Marker ist die linksventrikuläre Ejektionsfraktion (LVEF). Diese wurde in mehreren Studien evaluiert, deren Ergebnisse in einer Metaanalyse zusammengefasst wurden (Bailey et al. 2001). Eine eingeschränkte LVEF von 30-40 \% hatte in dieser Metaanalyse mit 7.294 Postmyokardinfarktpatienten eine Sensitivität von 59,1\% und eine Spezifität von 77,8 \% zur Vorhersage lebensbedrohlicher ventrikulärer Arrhythmien. Das relative Risiko (RR) betrug 4,3. Ein wesentlicher Nachteil für eine genauere Risikoabschätzung besteht allerdings darin, dass eine eingeschränkte LVEF nicht nur ein Risikoparameter für den SCD, sondern auch für die Gesamtmortalität darstellt, welche sich ab einer LVEF $<40$ \% exponentiell erhöht (Buxton et al. 2002; Buxton 2005; Buxton 2009).

Ein weiteres Risikostratifikationsverfahren ist die invasive elektrophysiologische Untersuchung (EPU). Ihre Durchführung wird in den aktuellen ACC/AHA-Leitlinien bei Patienten mit einer unklaren Synkope oder bei Postmyokardinfarktpatienten mit einer LVEF $\leq$ $40 \%$ und nicht-anhaltenden ventrikulären Tachykardien empfohlen (Epstein et al. 2008). In 
diesem Verfahren werden über einen intravasalen Zugang mehrere Elektrodenkatheter meist in das rechte Herz positioniert, um die Induzierbarkeit ventrikulärer Tachykardien (VT) mittels elektrischer Stimulation zu überprüfen. Die Induktion von VTs während der EPU ist mit einem erhöhten SCD-Risiko assoziiert. In einer Metaanalyse zur Evaluation dieses Verfahrens mit 4.022 Postmyokardinfarktpatienten betrug die Sensitivität $61,6 \%$, die Spezifität 84,1 \% und das RR 6,6 (Bailey et al. 2001).

Neben diesen etablierten Verfahren stehen mit der Spät-Potential-Analyse im signalgemittelten Elektrokardiogramm (El-Sherif et al. 1990; Gardner et al. 1985), der T-WellenAlternans-Untersuchung (Bloomfield et al. 2006; Costantini et al. 2009; Gehi et al. 2005; Rosenbaum et al. 1994) der QRS-Dauer-Bestimmung im Ruhe-Elektrokardiogramm (Baldasseroni et al. 2002; Kurl et al. 2012; Padmanabhan et al. 2003) oder der Bestimmung der Barorezeptorsensibilität (La Rovere et al. 2001) weitere diagnostische Verfahren zur Verfügung, deren prädiktiver Wert für die Risikostratifikation des SCDs in klinischen Studien überprüft wird.

Ein weiteres Verfahren dessen Rolle für die Risikostratifikation des SCDs noch nicht abschließend bewertet wurde, stellt die Bestimmung verschiedener Risikomarker aus dem 24h-Langzeit-Elektrokardiogramm dar.

\subsection{Risikostratifizierungsparameter im 24-h-Langzeit-Elektrokardiogramm}

Als Langzeit-Elektrokardiogramm (LZ-EKG) wird die Aufzeichnung eines Elektrokardiogramms über einen Zeitraum von meist 24 Stunden bezeichnet. Das LZ-EKG wird über zwei bis sechs auf den Brustkorb geklebte Elektroden kontinuierlich auf einen Recorder übertragen, der am Gürtel oder um den Hals getragen wird. Moderne Geräte speichern die gesammelten Daten digital auf Festplatten, Flash-Speicherkarten oder vergleichbaren Speichermedien. Nach Ende der Aufzeichnung werden die Daten computergestützt ausgewertet. Das konventionelle LZ-EKG dient hauptsächlich der Erkennung und Quantifizierung von Herzrhythmusstörungen bei Patienten mit einer bekannten Herzerkrankung oder mit Symptomen, die durch Rhythmusstörungen verursacht sein könnten. Dazu zählen Herzrasen, Herzstolpern, Schwindelattacken, Ohnmachtsanfälle und vorübergehende Lähmungserscheinungen (TIA). Es wurden verschiedene, im Folgenden näher erläuterte Marker zur Risikostratifikation durch das LZ-EKG entwickelt (Goldberger et al. 2008). 


\subsubsection{Ventrikuläre Extrasystolen}

Eine ventrikuläre Extrasystole (VES) ist eine spontane elektrische Erregung der Kammermuskulatur, die sich eigenständig, ohne Einfluss der vorangestellten Strukturen des Reizleitungssystems, aus einem ektopen Zentrum der Kammermuskulatur entwickelt hat. Folgen drei oder mehr VES aufeinander und liegt zudem eine Herzfrequenz $>100 /$ min vor, so besteht eine ventrikuläre Tachykardie (VT). Eine nicht-anhaltende ventrikuläre Tachykardie (nsVT) ist definiert durch eine maximale Anzahl von fünf aufeinanderfolgenden VES sowie eine Dauer von maximal $29 \mathrm{sec}$. Aufgrund des identischen Ursprunges werden VES oder nsVT auch unter dem Begriff der ventrikulären Ektopie zusammengefasst.

Ein erhöhtes Mortalitätsrisiko nach einem akuten Myokardinfarkt ist für Patienten mit einer vermehrten Anzahl von VES, auch ventrikuläre Extrasystolie genannt, oder einem Auftreten einer nsVT in größeren prospektiven Studien und Metaanalysen belegt (Bailey et al. 2001; Crawford et al. 1999; Denes et al. 1991; Goldberger et al. 2008; Maggioni et al. 1993; Scirica et al. 2010).

\subsubsection{Herzfrequenzvariabilität}

Die Herzfrequenzvariabilität (HRV) wird bedingt durch die Fluktuation der Herzfrequenz. Das physiologische Korrelat ist die vom Sinusknoten bestimmte Grundfrequenz, die vom autonomen Nervensystem je nach Bedarf moduliert wird. Eine hohe HRV spricht für eine gute Anpassungsfähigkeit des Organismus sowie für eine ausreichende Entlastungs- und Erholungsmöglichkeit des Herzens. Eine eingeschränkte HRV stellt eine Störung im Gleichgewicht des autonomen Nervensystems durch einen gesteigerten Sympathikotonus und einen verminderten Vagotonus dar. Dieses gestörte Gleichgewicht wird als Auslöser lebensbedrohlicher Herzrhythmusstörungen betrachtet (Schwartz et al. 1992; Vanoli et al. 1991). Ein erhöhtes SCD-Risiko für Postmyokardinfarktpatienten mit einer niedrigeren HRV als die jeweilige Kontrollgruppe ist durch mehrere Studien belegt (Bailey et al. 2001; Crawford et al. 1999; Farrell et al. 1991; La Rovere et al. 1998; La Rovere et al. 2001). Die Grenzwerte, die zwischen einer normalen und einer pathologischen HRV trennen, variieren je nach Studie. Definierte Normwerte der HRV-Bestimmung mittels des LZ-EKGs liegen aktuell nicht vor.

Es wurden verschiedene Verfahren zur Messung der HRV entwickelt (Ewing et al. 1984; TASK 1996). Die zeitbezogene und die frequenzbezogene HRV-Bestimmung sind die 
etabliertesten Verfahren, die untereinander eine hohe Korrelation aufweisen (Bigger et al. 1992b; TASK 1996). Die Berechnung der HRV nach diesen Verfahren wird im MethodenTeil näher erläutert.

\subsubsection{Herzfrequenzturbulenz}

Die Herzfrequenzturbulenz (HRT) beschreibt das physiologische Phänomen von Fluktuationen der Herzfrequenz nach einer ventrikulären Extrasystole bei Sinusrhythmus (Bauer et al. 2008). Die Ursache dieser Fluktuationen ist noch nicht geklärt. Eine führende Hypothese besagt, dass eine kompensatorische Pause der Herzerregung nach einer ventrikulären Extrasystole einen kurzen arteriellen Blutdruckabfall bewirkt, der über Barorezeptoren zu einer Herzfrequenzbeschleunigung führt (Jalife et al. 1983; Smith et al. 1995; Welch et al. 1989). Diese Herzfrequenzbeschleunigung verursacht nachfolgend über den gleichen Regelkreis in gegenläufiger Richtung eine ausgleichende Herzfrequenzentschleunigung. Damit stellt die HRT wie auch die HRV ein Maß für die Funktion des autonomen Nervensystems des Herzens dar.

Die Herzfrequenzbeschleunigung und die Geschwindigkeit der darauffolgenden Entschleunigung lassen sich numerisch quantifizieren (Bauer et al. 2008). Dabei stellt der Turbulenzbeginn (TO) ein Maß für die initiale Herzfrequenzbeschleunigung im Verhältnis zur Herzfrequenz in den Schlägen vor der ventrikulären Extrasystole dar. Der TO wird in Prozent gemessen. Eine physiologische Herzfrequenzbeschleunigung besteht bei einem TO $<0 \%$. Die Turbulenzsteigung (TS) ist ein Maß für die Geschwindigkeit der nachfolgenden Herzfrequenzabnahme (Bauer et al. 2008). Sie wird in Millisekunde pro Herzschlag-Intervall (ms/RR-Intervall) gemessen. Eine $\mathrm{TS}>2,5 \mathrm{~ms} / \mathrm{RR}$-Intervall stellt eine physiologische Reaktion dar.

Die Entwickler dieses Risikostratifizierungsverfahrens konnten zeigen, dass sich das Risiko exakter prognostizieren lässt, wenn beide Parameter kombiniert und $\mathrm{zu}$ insgesamt drei Kategorien zusammengefasst werden: Bei physiologischen Werten beider Parameter besteht das niedrigste Risiko, die sog. HRT-Kategorie 0. Die HRT-Kategorie 1 liegt bei einem pathologischen Wert einer der beiden Parameter vor und die HRT-Kategorie 2 bei pathologischen Werten beider Parameter (Bauer et al. 2008).

Eine verminderte HRT zeigte in größeren retrospektiven und prospektiven Studien mit Postmyokardinfarktpatienten sowohl eine erhöhte Gesamtmortalität als auch ein erhöhtes 
SCD-Risiko an (Barthel et al. 2003; Exner et al. 2007; Ghuran et al. 2002; Hallstrom et al. 2005; Makikalio et al. 2005; Schmidt et al. 1999).

\subsubsection{Dezelerations- und Akzelerationskapazität}

Mit der phasengleichgerichteten Signalmittelung (PRSA) ist eine neue Methode entwickelt worden, die periodisches und quasi-periodisches Verhalten in biologischen Signalen erkennt und begleitende Artefakte herausfiltert (Bauer et al. 2006b). Der Algorithmus trennt zwischen Herzfrequenzabnahmen und Herzfrequenzzunahmen als Unterscheidung zwischen den parasympathischen und sympathischen Einflüssen des autonomen Nervensystems. Der parasympathische Einfluss wird mittels der Dezelerationskapazität (DC) und der sympathische Einfluss mittels der Akzelerationskapazität (AC) dargestellt. Die Einheit der DC und AC ist Millisekunde (ms).

Eine erhöhte Gesamtsterblichkeit für Postmyokardinfarktpatienten bei einer erniedrigten DC oder erhöhten AC konnte in einer großen multizentrischen prospektiven Studie mit 2.711 Teilnehmern nachgewiesen werden (Bauer et al. 2006a). Für die DC erfolgt eine Unterteilung in Kategorien mit einem hohen Risiko ( $\mathrm{DC} \leq 2,5 \mathrm{~ms}$ ), einem intermediären ( $\mathrm{DC}>2,5$ $\leq 4,5 \mathrm{~ms}$ ) und einem geringen Risiko $(>4,5 \mathrm{~ms})$. Eine ähnliche Grenzwertbestimmung für die AC wurde nicht vorgenommen.

\subsection{Fragestellung und Zielsetzung}

Im Rahmen der vorliegenden Arbeit soll untersucht werden, ob folgende Parameter aus dem LZ-EKG das Auftreten appropiater Schockabgaben und die Gesamtmortalität bei Patienten nach Erstimplantation eines ICDs vorhersagen können:

- Quantifizierung ventrikulärer Extrasystolen und nicht-anhaltender ventrikulärer Tachykardien

- Herzfrequenzvariabilität

- Herzfrequenzturbulenz

- Dezelerations- und Akzelerationskapazität 


\section{Methode}

\subsection{Studienbeschreibung}

Die vorliegende Arbeit ist eine prospektive Beobachtungsstudie an Patienten, bei denen im Zeitraum vom 1. Juni 2008 bis zum 31. November 2009 aus primärprophylaktischer oder sekundärprophylaktischer Indikation im Herzzentrum der Universitätsmedizin Göttingen eine ICD-Neuimplantation erfolgte. Diese Patienten unterzogen sich periinterventionell einer LZEKG-Untersuchung, welche anschließend computergestützt ausgewertet wurde (siehe Kapitel 2.3). In der Auswertung dieser LZ-EKG-Aufzeichnungen erfolgte die Quantifizierung ventrikulärer Extrasystolen, nicht-anhaltender ventrikulärer Tachykardien, die Bestimmung verschiedener Parameter der Herzfrequenzvariabilität, der Herzfrequenzturbulenz sowie der Dezelerations- und Akzelerationskapazität. In der Nachbeobachtung wurden zudem die Mortalität sowie das Auftreten des ersten appropiaten ICD-Schocks erfasst (siehe Kapitel 2.7).

Die Studie erfolgte im Rahmen eines Projektes mit weiteren Studien, die verschiedene nicht invasive SCD-Risikostratifikationsverfahren wie z. B. die T-Wellen-Alternans-Untersuchung oder die Spät-Potential-Analyse im signal-gemittelten Elektrokardiogramm untersuchten. Den Patienten wurde zu Beginn die Durchführung und der Zweck der nicht-invasiven Risikostratifikationsverfahren, die Speicherung der Daten sowie die Teilnahme an Verlaufskontrollen im ärztlichen Gespräch erläutert. Die schriftliche Aufklärung erfolgte mittels eines Infoblattes. Das Einverständnis des Patienten wurde schriftlich festgehalten und konnte zu jedem Studienzeitpunkt widerrufen werden.

\subsection{Ein- und Ausschlusskriterien}

Das wichtigste Einschlusskriterium war die ICD-Erstimplantation aus primärprophylaktischer oder sekundärprophylaktischer Indikation nach aktuellen Leitlinien (Epstein et al. 2008). Patienten, die zum Zeitpunkt der Implantation das 18. Lebensjahr noch nicht vollendet hatten oder sich in Betreuung der kinderkardiologischen Abteilung der Universitätsmedizin Göttingen befanden, wurden nicht in die Studie aufgenommen. Zur Festlegung eines gemeinsamen Ausgangspunktes sowie zur kontinuierlichen Überwachung der Postimplantationsphase wurden nur Neuimplantationen beobachtet. Patienten, bei denen ein Aggregatwechsel, eine Schrittmacher-Aufrüstung zum ICD oder die Revision eines bestehenden ICD-Systems erfolgte, wurden nicht in die Studie aufgenommen. 
Die Berechnungen der HRV, HRT, AC und DC setzen einen Sinusrhythmus voraus. Patienten, die im routinemäßig bei Aufnahme durchgeführten 12-Kanal-Ruhe-EKG ein Vorhofflimmern oder Vorhofflattern aufwiesen, wurden deswegen nicht in die Studie aufgenommen.

Eine LZ-EKG-Aufzeichnung wurde dann als valide bewertet, wenn in dieser für mindestens 10 Stunden ein Sinusrhythmus mit verwertbarer Qualität aufgezeichnet wurde.

Patienten, die eine Teilnahme an der Studie initial ablehnten oder später ihre Einwilligung widerriefen, wurden nicht in das Studienregister aufgenommen bzw. nachträglich aus diesem entfernt.

\subsection{Computergestützte Auswertung}

Die aufgezeichneten LZ-EKGs wurden mit einer halbautomatischen Auswertungs-Software (Pathfinder, Version 8.602, Delmar Reynolds/Spacelabs Healthcare, Washington, USA) und durch eine anschließende manuelle Korrektur ausgewertet. Abbildung 1 zeigt einen Ausschnitt aus dem Analysemenü des Pathfinder-Programmes. Die Analyse erfolgte unter Bewertung drei verschiedener Ableitungen. Die Länge der Intervalle wurde anhand der Abstände der R-Zacken (sog. RR-Intervalle) ermittelt und in Millisekunden bestimmt. Intervalle zwischen benachbarten QRS-Komplexen, die in der Auswertung als normal bewertet wurden und somit jeweils aus einer Depolarisation des Sinusknoten resultierten, wurden als sog. NN-Intervalle (Normal-zu-Normal-Intervall) klassifiziert. Intervalle zwischen einem normalen QRS-Komplex und einem als aberrant befundeten QRS-Komplex (z. B. eine VES oder ein ventrikulärer Herzschrittmacherimpuls) wurden als NA-Intervalle (Normal-zuAberrant-Intervall) bzw. bei zwei benachbarten aberranten QRS-Komplexen als AAIntervalle (Aberrant-zu-Aberrant-Intervall) klassifiziert.

Das Pathfinder-Programm erstellte einen Datensatz, in dem die Informationen über die Intervalllängen zwischen den benachbarten QRS-Komplexen, deren Reihenfolge und die Bewertung der QRS-Komplexe (normal oder aberrant) gespeichert wurden.

Dieser Datensatz diente zur Weiterverarbeitung durch eine spezielle Auswertungs-Software. So erfolgte die Berechnung der Parameter der HRT, der DC und der AC anhand der erstellten Datensätze durch das Programm Librasch Calc (Version 1.02, geschrieben von Raphael Schneider TU München, Deutschland) nach standardisierten Methoden (Bauer et al. 2006b; Bauer et al. 2008). Die im folgenden Kapitel erläuterten Parameter der frequenzbezogenen 
HRV wurden durch das Programm HRV-Tools (Version 1.74, Delmar Reynold/Spacelabs Healthcare, Washington, USA) bestimmt.

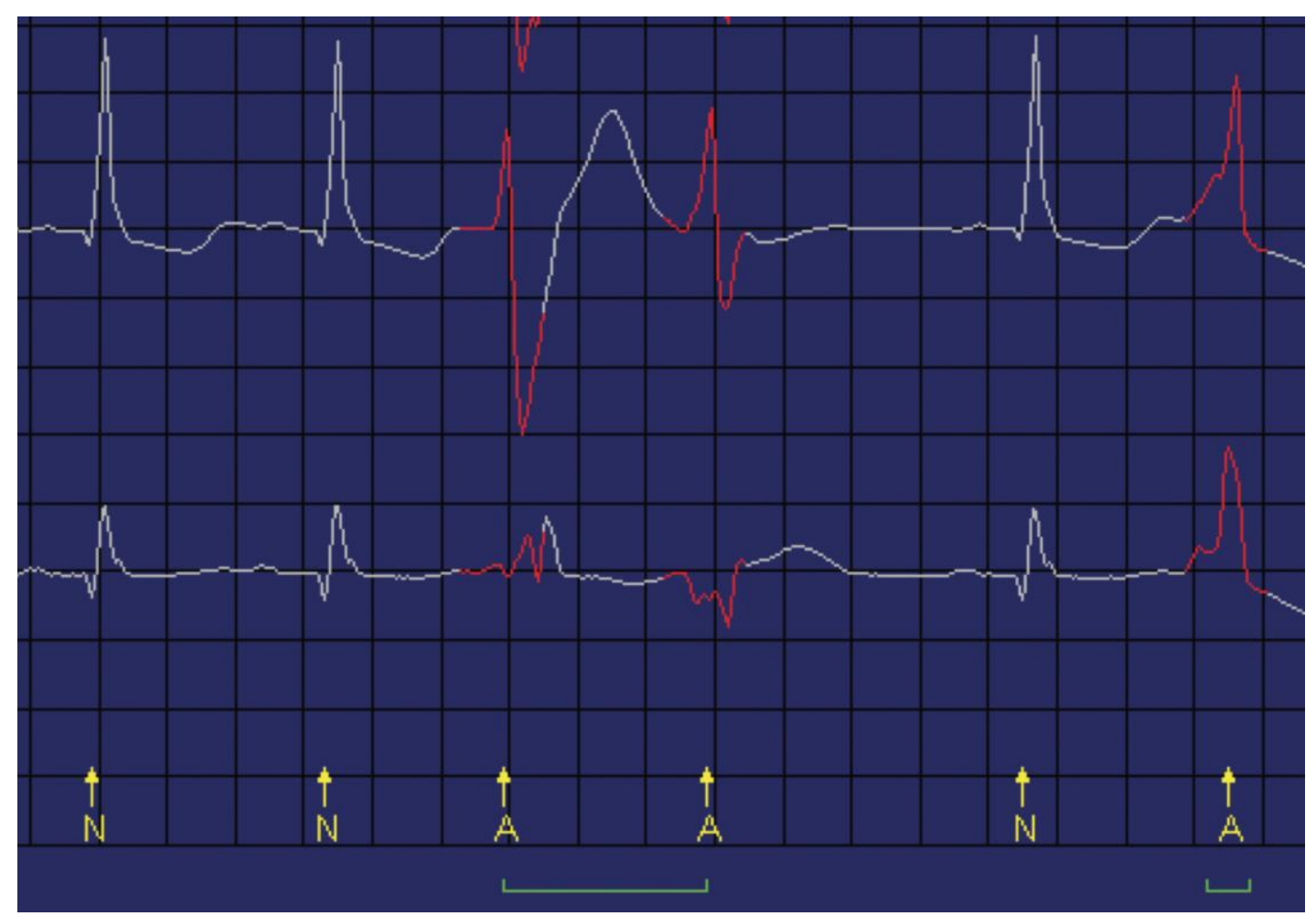

Abb. 1 Ausschnitt aus dem Analysemenü des Pathfinder-Programmes

\subsection{Berechnung der Herzfrequenzvariabilität}

Für die Bestimmung der HRV existieren mehrere standardisierte Methoden (TASK 1996). Die für diese Arbeit durchgeführten zeitbezogenen und frequenzbezogenen Messungen der HRV stellen die etabliertesten Verfahren dar. Die Berechnung der HRV erfolgte anhand der Zeitreihen der NN-Intervalle in den Datensätzen, die nach halbautomatischer Auswertung durch das Pathfinder-Programm erstellt wurden. RR-Intervalle, die aberrante QRS-Komplexe enthielten, wurden somit von der Berechnung ausgeschlossen.

Es wurden folgende statistische Parameter der zeitbezogenen HRV durch das PathfinderProgramm berechnet:

- SDNN (engl. standard deviation of NN): Standardabweichung aller NN-Intervalle

- SDNNi (SDNN-Index): Mittelwert der Standardabweichungen aller in fünf-minütige Abschnitte unterteilten NN-Intervalle der gesamten Aufzeichnungsdauer 
- SNN50+: Anzahl aller NN-Intervalle, die eine Größenänderung von über $50 \mathrm{~ms}$ im Vergleich zum vorausgehenden NN-Intervall aufweisen

- RMSSD (engl. root mean square of successive differences between adjacent NNintervals): Quadratwurzel des Mittelwertes der Summe der quadrierten Differenzen aufeinanderfolgender NN-Intervalle

Die mathematische Grundlage zur Berechnung der frequenzbezogenen HRV durch das für diese Arbeit verwendete Programm HRV-Tools ist das Verfahren der schnellen FourierTransformation (Oppenheim und Schafer 1975). Dieses Verfahren bietet die Möglichkeit, verschiedene wiederkehrende Änderungen der Herzfrequenz in Frequenzbereiche zusammenzufassen (Akselrod et al. 1981). Diese Frequenzbereiche werden unterschiedlichen Einflüssen des autonomen Nervensystems zugeordnet (Akselrod et al. 1981; TASK 1996). Es wurden für diese Arbeit folgende Parameter der frequenzbezogenen HRV berechnet:

- HF (engl. high frequency): Band der hohen Frequenzen (Frequenzbereich: 0,15 bis 0,4 Hz); Messwert der parasympathischen Regulation der Herzfrequenz (Einheit $\mathrm{ms}^{2}$ )

- LF (engl. low frequency): Band der niedrigen Frequenzen (Frequenzbereich 0,05 bis $0,15 \mathrm{~Hz}$ ); Messwert der sympathischen und parasympathischen Regulation der Herzfrequenz (Einheit $\mathrm{ms}^{2}$ )

- VLF (engl. very low frequency): Band der sehr niedrigen Frequenzen (Frequenzbereich: 0,0033 bis 0,04 Hz); nach einer umstrittenen Hypothese ein Messwert für humorale Sinusknotenmodulationen durch Katecholamine oder das Renin-Angiotensin-Aldosteron-System (Einheit $\mathrm{ms}^{2}$ )

- LF/HF-Quotient: Messwert für die Balance zwischen sympathischer und parasympathischer Regulation der Herzfrequenz

Da verschiedene Analyse-Programme mit unterschiedlichen Einstellungen eine HRVBerechnung durchführen, wird zur Vergleichbarkeit eine exakte Beschreibung der verwendeten Einstellungen gefordert (TASK 1996). Bei dem für diese Arbeit verwendeten Programm betrug die Dauer eines Abtastungsintervalls jeweils fünf Minuten und pro Intervall wurden 2.048 Abtastungspunkte bestimmt. Die nachfolgende Interpolation wurde mit der Cubic Spline-Methode durchgeführt und die Abtastrate nach Interpolation betrug $4 \mathrm{~Hz}$. Die Fenstermethode erfolgte nach Hamming und zur Mittelung wurde die Welch-Methode angewandt. Das Programm berechnete anschließend Mittelwerte für die gesamte Aufzeichnungsdauer. 


\subsection{Endpunktbeschreibung und Nachsorge}

Vor Beginn der Studie wurden folgende Endpunkte definiert:

- Gesamtmortalität

- Auftreten der ersten appropiaten ICD-Schockabgabe

- Kombinierter Endpunkt (Gesamtmortalität und Auftreten der ersten appropiaten ICDSchockabgabe)

Die Nachbeobachtung erfolgte im Rahmen der Nachsorge durch die ICD-Ambulanz des Herzzentrums Göttingen. Dort wurden die Patienten routinemäßig in drei- bis sechsmonatigen Abständen einbestellt und die Speicher der ICDs ausgelesen. Ein ICD dokumentiert in diesem Speicher Informationen über detektierte Rhythmusstörungen sowie der ggf. durchgeführten Therapie. Bei Patienten, deren Nachsorge nicht in der ICD-Ambulanz des Herzzentrums Göttingen durchgeführt wurde, wurden entsprechende Befunde vom weiterbehandelnden Kardiologen oder Hausarzt angefordert.

\subsection{Statistische Analyse}

Die Berechnung der grundlegenden statistischen Kenngrößen (Mittelwert, Standardabweichung und Median) erfolgte mit dem Tabellenkalkulationsprogramm Excel $2011^{\mathrm{TM}}$ der Firma Microsoft Corporation.

Zur Bestimmung der unabhängigen Korrelation zwischen dem Ergebnis der Bestimmung der einzelnen LZ-EKG-Risikomarker und dem Erreichen eines der Endpunkte wurde das statistische Verfahren der Cox-Regression angewandt. Dieses Verfahren wurde gewählt, da es die verschiedenen Zeitpunkte der ICD-Implantationen und die daraus resultierenden unterschiedlichen Nachbeobachtungszeiten berücksichtigt. Zur Durchführung der CoxRegression wurde das Statistikprogramm SPSS Statistics ${ }^{\text {TM }}$ Version 22 der Firma IBM Corporation verwendet.

Die Kategorisierung der im Cox-Regressionsmodell zu vergleichenden Gruppen erfolgte nach den aus der Literatur bekannten Grenzwerten der einzelnen LZ-EKG-Risikomarker. Da einheitliche Grenzwerte für die ventrikuläre Extrasystolie, die Parameter der Herzfrequenzvariabilität sowie für die Akzelerationskapazität fehlen, erfolgte für diese LZEKG-Risikomarker als objektivster Grenzwert eine Dichotomisierung anhand des Medians. 
Die Analyse erfolgte sowohl in einem univariaten Cox-Regressionsmodell als auch in einem multivariaten Cox-Regressionsmodell mit den Ko-Variablen Patientenalter und Geschlecht.

Als Kriterium für statistische Signifikanz wurde eine Irrtumswahrscheinlichkeit $\leq 5 \%$ (pWert $\leq 0,05)$ festgelegt. Das $95 \%$-Konfidenzintervall der bestimmten Hazard-Rate durfte den Wert 1 nicht beinhalten.

Der zeitliche Verlauf des endpunktfreien Überlebens wurde graphisch mittels Kaplan-MeierÜberlebenskurve dargestellt. Dazu wurde ebenfalls das Statistikprogramm SPSS Statistics ${ }^{\text {TM }}$ Version 22 der Firma IBM Corporation verwendet.

\subsection{Ethik und Datenschutz}

Die erhobenen Patientendaten wurden auf einem geschützten Serverbereich gespeichert. Es wurde keine Bearbeitung und Speicherung außerhalb dieses Bereiches vorgenommen. Die statistische Analyse erfolgte anhand pseudonymisierter Daten. Die Durchführung der LZEKGs erfolgte aufgrund klinischer Indikation nach ärztlicher Anordnung. Die Beantragung eines Ethikvotums war nicht erforderlich. 


\section{Ergebnisse}

\subsection{Patientencharakteristika}

150 Patienten wurden unter Beachtung der Ein- und Ausschlusskriterien (vgl. Kapitel 2.2) rekrutiert. Die Basischarakteristika dieser Studienpatienten sind in Tabelle 1 dargestellt. Die ICD-Implantationen erfolgten überwiegend aus primärprophylaktischer Indikation (66 \%) bei einer hochgradigen Einschränkung der LVEF (mittlere LVEF: $32 \pm 11 \%$ ). Am häufigsten bestand als zugrundeliegende Grunderkrankung eine ischämische Kardiomyopathie (60\%). Die Mehrheit der Patienten nahmen ACE/AT1-Hemmer (91\%) und Betablocker (89\%) ein.

\begin{tabular}{|lc|}
\hline \multicolumn{2}{|c|}{ Tabelle 1 } \\
Charakteristika der Studienpatienten \\
\hline $\begin{array}{l}\text { Patientenanzahl } \\
\text { männlich } \\
\text { Alter }\end{array}$ & $\mathrm{n}=150$ \\
\hline ICD-Indikation: & $115(77 \%)$ \\
\hline Primärprophylaxe & $64 \pm 13 \mathrm{Jahre}$ \\
\hline Sekundärprophylaxe & \\
\hline Linksventrikuläre Ejektionsfraktion: & $99(66 \%)$ \\
\hline durchschnittliche linksventrikuläre Ejektionsfraktion & $51(34 \%)$ \\
\hline 35\% & \\
$>$ 35\% & $32 \pm 11 \%$ \\
Zugrundeliegende Herzerkrankung: & $116(77 \%)$ \\
\hline ischämische Kardiomyopathie & $34(23 \%)$ \\
- Z. n. Myokardinfarkt & \\
dilatative Kardiomyopathie & $90(60 \%)$ \\
arrhythmogene rechtsventrikuläre Kardiomyopathie & $68(45 \%)$ \\
hypertrophe Kardiomyopathie & $33(22 \%)$ \\
\hline Medikation: & $2(1 \%)$ \\
\hline Betablocker & $0(0 \%)$ \\
Schleifendiuretika & \\
ACE/AT1-Hemmer & $134(89 \%)$ \\
Aldosteron-Antagonist & $83(55 \%)$ \\
Amiodaron & $136(91 \%)$ \\
Digoxin & $77(51 \%)$ \\
\hline
\end{tabular}




\subsection{Patienten mit ICD-Neuimplantation ohne Aufnahme in das Studienregister}

Die Anzahl der ICD-Neuimplantationen im Rekrutierungszeitraum betrug 227, davon wurden 150 Patienten in die Studie aufgenommen. Die Aufnahme der übrigen 77 Patienten war aus unterschiedlichen Gründen nicht möglich. Der quantitativ bedeutsamste Faktor, der zum Ausschluss von 40 Patienten führte, war das Fehlen eines Sinusrhythmus. 20 LZ-EKGAufzeichnungen erfüllten die zu Studienbeginn definierten Validitätskriterien $(\geq 10$ Stunden Aufzeichnungsdauer mit $\geq 40 \%$ zur HRV-Berechnung geeigneter Daten) nicht. In 8 dieser Fälle verhinderte eine dominierende Herzschrittmacher-Aktivität die Einhaltung der Validitätskriterien. Andere Ursachen, die zur Verletzung der Validitätskriterien führten, waren z. B. die fehlende Compliance beim Tragen des LZ-EKG-Rekorders und durch Artefakte nicht bewertbare LZ-EKG-Aufzeichnungen.

Bei 9 Patienten gelang es aus organisatorischen Gründen vor allem zu Beginn der Rekrutierungsphase nicht, ein LZ-EKG durchzuführen. 5 Patienten lehnten initial oder im Verlauf die Studienteilnahme $a b$ und bei 2 Patienten gelang keine vollständige Nachbeobachtung.

\subsection{Nachbeobachtung}

Die mittlere Dauer der Nachbeobachtung betrug $28 \pm 12$ Monate. Die kürzeste Nachbeobachtung erfolgte an einem Patienten, der 10 Tage nach ICD-Implantation verstarb. Am längsten wurde eine Patientin beobachtet, die 45 Monate nach ICD-Implantation keinen der Endpunkte erreicht hatte.

In der Nachbeobachtungszeit verstarben 25 Patienten. Die jährliche Mortalitätsrate betrug 7,1 \%. Bei 20 Patienten löste der ICD mindestens einen appropiaten Schock aus. Die jährliche Schockrate betrug 5,7 \% und die Zahl der ICD-Implantationen pro Patient mit appropiatem ICD-Schock 7,5. 4 Patienten, bei denen ein appropiater ICD-Schock ausgelöst wurde, verstarben während der Nachbeobachtungsphase. Der kombinierte Endpunkt aus Gesamtmortalität und ICD-Schock wurde somit von 41 Patienten (27 \%) erreicht. 


\subsection{Prävalenz klinischer Ereignisse in den LZ-EKG-Risikomarkergruppen}

Zur Vorbereitung auf die weitere Analyse erfolgte eine Eingruppierung der Studienpatienten nach dem Ergebnis des jeweiligen LZ-EKG-Risikomarkers. Soweit vorhanden, wurden die dazu aus der Literatur bekannten Grenzwerte verwandt. Bei fehlenden einheitlichen Literaturangaben erfolgte eine Dichotomisierung anhand des Medians (vgl. Tabelle 2).

Die Mehrzahl der verstorbenen Patienten (72\%), der Patienten mit einem appropiaten ICDSchock (70 \%) und der Patienten, die den kombinierten Endpunkt erreichten (68\%) wiesen eine ventrikuläre Extrasystolie auf. Bei den Patienten, die keinen der Endpunkte erreichten, bestand bei 43-47 \% - je nach Endpunkt - ebenfalls eine ventrikuläre Extrasystolie.

Bei den Patienten, die keinen der Endpunkte erreichten, wies die nsVT die geringste Prävalenz auf (21-24\%). Bei den Patienten, die einen der Endpunkte erreichten, konnte bei $39-40 \%$ eine nsVT nachgewiesen werden.

Die Herzfrequenzturbulenz war der einzige LZ-EKG-Risikomarker, der nicht bei allen Patienten bestimmt werden konnte, da sechs LZ-EKG-Aufzeichnungen die Kriterien zur validen HRT-Berechnung nicht erfüllten (Bauer et al. 2008). Keiner dieser fehlenden sechs Patienten erreichte einen der Endpunkte. 


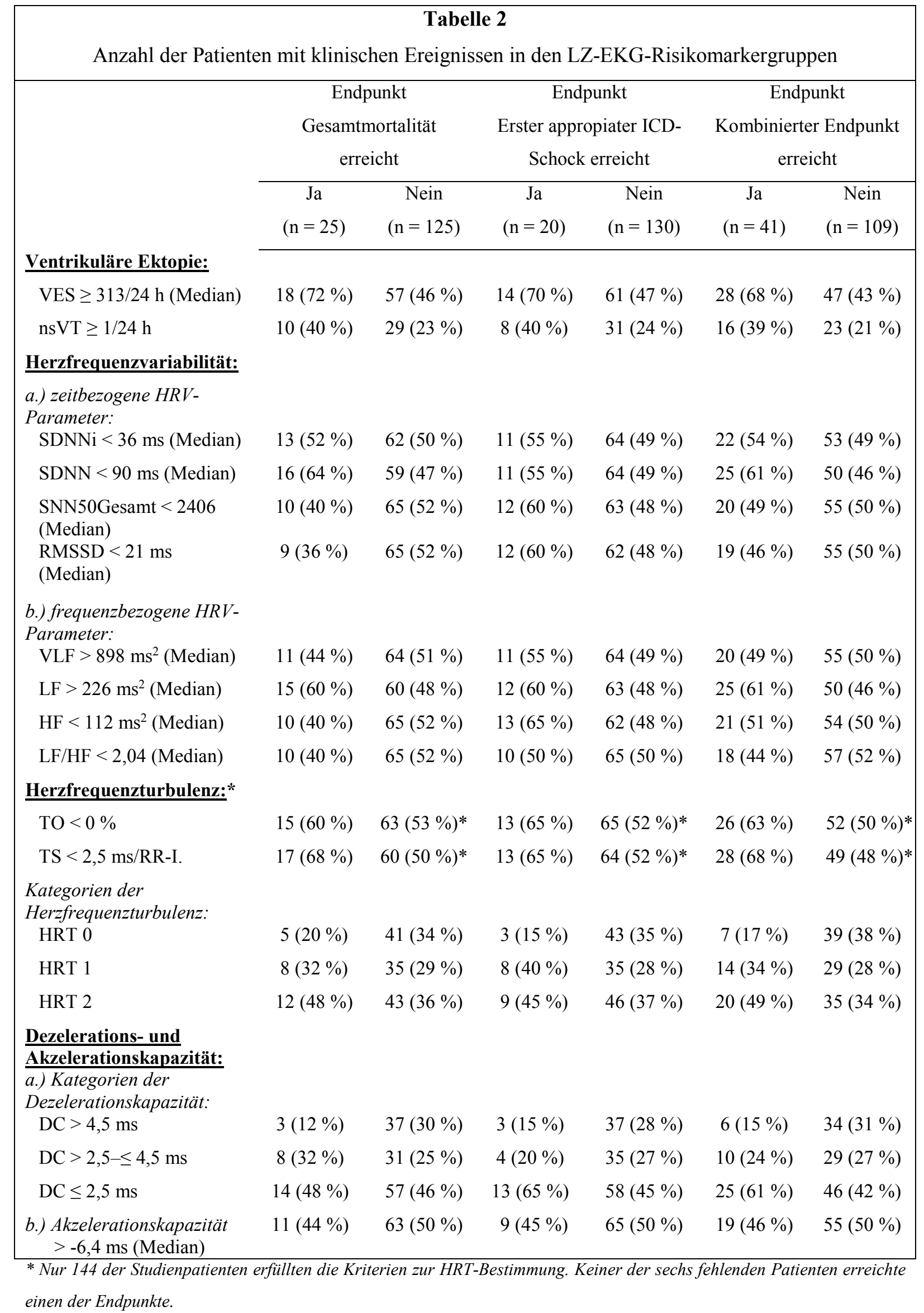




\subsection{Univariate Analyse zur Vorhersagbarkeit des Erreichens der Endpunkte}

Ein statisch signifikant erhöhtes Risiko für das Erreichen des Endpunktes Gesamtmortalität bestand in der univariaten Analyse nur für Patienten mit einer ventrikuläre Extrasystolie (vgl. Tabelle 3). Das Vorhandensein von $\geq 1 \mathrm{nsVT} / 24 \mathrm{~h}$, einem TS-Wert $<2,5 \mathrm{~ms} / \mathrm{RR}-\mathrm{I}$. oder einem SDNN-Wert $<90$ ms zeigte einen Trend auf $(\mathrm{p}$-Wert $\leq 0,1)$.

Für keinen der LZ-EKG-Risikomarker konnte eine statistisch signifikante Assoziation mit dem Auftreten eines appropiaten ICD-Schocks nachgewiesen werden. Das Vorliegen einer ventrikulären Extrasystolie verletzte knapp die Signifikanzkriterien (HR: 2,58; 95\%-KI: $0,99-6,71 ; \mathrm{p}=0,05)$. Das Vorhandensein von $\geq 1 \mathrm{nsVT} / 24 \mathrm{~h}$ und die Vergleiche der Kategorien HRT 2 und HRT 1 mit der Kategorie HRT 0 zeigten ebenfalls einen Trend für eine Korrelation mit dem Auftreten eines appropiaten ICD-Schocks.

Ein in der univariaten Analyse signifikant erhöhtes Risiko für das Erreichen des kombinierten Endpunktes aus appropiatem ICD-Schock und Gesamtmortalität zeigten beide LZ-EKGRisikomarker der ventrikulären Ektopie, ein TS-Wert $<2,5 \mathrm{~ms} / \mathrm{RR}-\mathrm{I}$. sowie die Kategorie HRT 2 und HRT 1 im Vergleich zur Kategorie HRT 0 an. Zwischen den Kategorien der Dezelerationskapazität bestand nur für Patienten mit einer DC $\leq 2,5 \mathrm{~ms}$ im Vergleich zu Patienten mit einer DC $>4,5 \mathrm{~ms}$ ein statistisch signifikant höheres Risiko für das Erreichen des kombinierten Endpunktes. Ein SDNN-Wert $<90 \mathrm{~ms}$ zeigte einen Trend für einen Zusammenhang mit dem Erreichen des kombinierten Endpunktes an. 


\begin{tabular}{|c|c|c|c|c|c|c|}
\hline \multicolumn{7}{|c|}{$\begin{array}{c}\text { Tabelle } 3 \\
\text { Univariate Hazard-Ratios und p-Werte }\end{array}$} \\
\hline & \multirow{2}{*}{\multicolumn{2}{|c|}{$\begin{array}{c}\text { Endpunkt } \\
\text { Gesamtmortalität }\end{array}$}} & \multicolumn{2}{|c|}{ Endpunkt } & \multirow{2}{*}{\multicolumn{2}{|c|}{$\begin{array}{c}\text { Endpunkt } \\
\text { Kombinierter Endpunkt }\end{array}$}} \\
\hline & & & Erster appropiater 1 & -Schock & & \\
\hline & HR $(95 \%-\mathrm{KI})$ & $\mathrm{p}$-Wert & HR $(95 \%-K I)$ & $\mathrm{p}$-Wert & HR $(95 \%-\mathrm{KI})$ & $\mathrm{p}$-Wert \\
\hline \multicolumn{7}{|l|}{ Ventrikuläre Ektopie: } \\
\hline VES $\geq 313 / 24 \mathrm{~h}$ (Median) & $2,7(1,12-6,49)$ & 0,03 & $2,58(0,99-6,71)$ & 0,05 & $2,4(1,24-4,65)$ & $<0,01$ \\
\hline $\mathrm{nsVT} \geq 1 / 24 \mathrm{~h}$ & $1,98(0,89-4,42)$ & 0,1 & $2,21(0,9-5,41)$ & 0,08 & $2,12(1,13-3,97)$ & 0,02 \\
\hline \multicolumn{7}{|l|}{$\begin{array}{l}\text { Herzfrequenzvariabilität: } \\
\text { a.) zeitbezogene } H R V \text {-Parameter: }\end{array}$} \\
\hline $\mathrm{SDNNi}<36 \mathrm{~ms}$ (Median) & $1,11(0,51-2,45)$ & 0,79 & $1,19(0,49-2,87)$ & 0,7 & $1,16(0,63-2,14)$ & 0,64 \\
\hline SDNN < $90 \mathrm{~ms}$ (Median) & $1,98(0,88-4,48)$ & 0,1 & $1,36(0,56-3,29)$ & 0,5 & $1,75(0,93-3,28)$ & 0,08 \\
\hline SNN50Gesamt $<2406$ (Median) & $0,67(0,30-1,5)$ & 0,34 & $1,53(0,63-3,75)$ & 0,35 & $0,97(0,53-1,79)$ & 0,92 \\
\hline RMSSD $<21$ ms (Median) & $0,56(0,25-1,27)$ & 0,16 & $1,5(0,61-3,68)$ & 0,37 & $0,87(0,47-1,6)$ & 0,64 \\
\hline \multicolumn{7}{|l|}{ b.) frequenzbezogene $H R V$-Parameter: } \\
\hline VLF $>898 \mathrm{~ms}^{2}$ (Median) & $0,76(0,34-1,67)$ & 0,49 & $1,14(0,47-2,75)$ & 0,77 & $0,9(0,49-1,67)$ & 0,74 \\
\hline $\mathrm{LF}>226 \mathrm{~ms}^{2}$ (Median) & $0,62(0,28-1,39)$ & 0,24 & $0,64(0,26-1,56)$ & 0,32 & $1,67(0,89-3,13)$ & 0,11 \\
\hline $\mathrm{HF}<112 \mathrm{~ms}^{2}$ (Median) & $0,63(0,28-1,4)$ & 0,26 & $1,85(0,74-4,65)$ & 0,19 & $1,03(0,56-1,9)$ & 0,93 \\
\hline $\mathrm{LF} / \mathrm{HF}<2,04$ (Median) & $0,62(0,28-1,38)$ & 0,24 & $0,95(0,4-2,29)$ & 0,91 & $0,73(0,39-1,35)$ & 0,32 \\
\hline \multicolumn{7}{|l|}{ Herzfrequenzturbulenz: } \\
\hline $\mathrm{TO}<0 \%$ & $1,3(0,58-2,9)$ & 0,52 & $1,72(0,69-4,33)$ & 0,25 & $1,59(0,84-3)$ & 0,15 \\
\hline $\mathrm{TS}<2,5 \mathrm{~ms} / \mathrm{RR}-\mathrm{I}$. & $2,03(0,88-4,72)$ & 0,1 & $1,89(0,75-4,74)$ & 0,17 & $2,22(1,15-4,29)$ & 0,02 \\
\hline \multicolumn{7}{|l|}{ Kategorien der Herzfrequenzturbulenz: } \\
\hline HRT 1 vs. HRT 0 & $1,85(0,6-5,65)$ & 0,28 & $3,28(0,87-12,35)$ & 0,08 & $2,53(1,02-6,25)$ & 0,05 \\
\hline HRT 2 vs. HRT 0 & $2,17(0,77-6,17)$ & 0,15 & $3(0,81-11,11)$ & 0,1 & $2,86(1,21-6,76)$ & 0,02 \\
\hline HRT 2 vs. HRT 1 & $1,17(0,48-2,88)$ & 0,73 & $1,07(0,42-2,86)$ & 0,86 & $1,13(0,57-2,24)$ & 0,72 \\
\hline \multicolumn{7}{|l|}{ Dezelerations- und Akzelerationskapazität: } \\
\hline \multicolumn{7}{|l|}{ Kategorien der Dezelerationskapazität: } \\
\hline $\mathrm{DC} \leq 2,5 \mathrm{~ms}$ vs. $\mathrm{DC}>2,5-\leq 4,5 \mathrm{~ms}$ & $2,62(0,69-9,9)$ & 0,16 & $1,34(0,3-5,99)$ & 0,7 & $1,63(0,59-4,48)$ & 0,34 \\
\hline $\mathrm{DC} \leq 2,5 \mathrm{~ms}$ vs. $\mathrm{DC}>4,5 \mathrm{~ms}$ & $2,7(0,77-9,35)$ & 0,12 & $2,73(0,78-9,62)$ & 0,12 & $2,83(1,08-6,41)$ & 0,03 \\
\hline $\mathrm{DC}>2,5-\leq 4,5 \mathrm{~ms}$ vs. $\mathrm{DC}>4,5 \mathrm{~ms}$ & $1,03(0,43-2,46)$ & 0,95 & $2,04(0,67-6,29)$ & 0,21 & $1,61(0,77-3,37)$ & 0,2 \\
\hline Akzelerationskapazität > -6,4 ms (Median) & $0,76(0,35-1,69)$ & 0,5 & $1,31(0,54-3,17)$ & 0,55 & $0,79(0,43-1,46)$ & 0,45 \\
\hline
\end{tabular}

Fett-Kursiv: statistisch signifikante Risikomarker 


\subsection{Multivariate Analyse zur Vorhersagbarkeit des Erreichens der Endpunkte}

In der multivariaten Analyse unter Einschluss der Ko-Variablen Alter und Geschlecht wies keiner der bestimmten LZ-EKG-Risikomarker eine signifikante Korrelation mit dem Erreichen des Endpunktes Gesamtmortalität auf (vgl. Tabelle 4).

Ein statistischer Trend für eine unabhängige Assoziation mit dem Endpunkt des ersten appropiaten ICD-Schocks bestand für die Risikomarker ventrikuläre Extrasystolie (HR: 2,58; 95 \%-KI: 0,95-6,99; $\mathrm{p}=0,06)$, dem Vorhandensein von $\geq 1 \mathrm{nsVT} / 24 \mathrm{~h}(\mathrm{HR}: 2,17 ; 95$ \%-KI: 0,87-5,38; $\mathrm{p}=0,1$ ) und im Vergleich zwischen den Kategorien HRT 1 und HRT 0 (HR: 3,36; $95 \%-\mathrm{KI}: 0,85-13,32 ; \mathrm{p}=0,09)$.

Das Vorhandensein von $\geq 1 \mathrm{nsVT} / 24 \mathrm{~h}$ zeigte als einziger LZ-EKG-Risikomarker in der multivariaten Analyse statistisch signifikant ein höheres Risiko für das Erreichen des kombinierten Endpunktes an. In der graphischen Analyse mittels Kaplan-MeierÜberlebenskurve deutet sich diese prognostische Relevanz vor allem durch Ereignisse innerhalb der ersten zehn Monate nach ICD-Implantation an (vgl. Abb. 2). Der Risikomarker ventrikuläre Ektopie wies durch mathematische Rundung einen p-Wert von 0,05 auf, verletzte jedoch nach Berechnung des Konfidenzintervalls knapp die definierten Signifikanzkriterien (HR: 1,96; 95 \%-KI: 0,99-3,89). Für einen TS-Wert <2,5 ms/RR-I. und in den Vergleichen der Kategorien HRT 2 und HRT 1 mit der Kategorie HRT 0 konnte ebenfalls ein Trend für eine Korrelation mit dem Erreichen des kombinierten Endpunktes aufgezeigt werden. 




Abb. 2 Ereignisfreies Überleben für den kombinierten Endpunkt aus appropiatem ICD-Schock und Gesamtmortalität in Abhängigkeit vom Ergebnis der nsVT-Quantifizierung $(p=0,05)$ 


\begin{tabular}{|c|c|c|c|c|c|c|}
\hline \multicolumn{7}{|c|}{$\begin{array}{c}\text { Tabelle } 4 \\
\text { Multivariate Hazard-Ratios und p-Werte }\end{array}$} \\
\hline & \multirow{2}{*}{\multicolumn{2}{|c|}{$\begin{array}{c}\text { Endpunkt } \\
\text { Gesamtmortalität }\end{array}$}} & \multirow{2}{*}{\multicolumn{2}{|c|}{$\begin{array}{c}\text { Endpunkt } \\
\text { Erster appropiater ICD-Schock }\end{array}$}} & \multirow{2}{*}{\multicolumn{2}{|c|}{$\begin{array}{c}\text { Endpunkt } \\
\text { Kombinierter Endpunkt }\end{array}$}} \\
\hline & & & & & & \\
\hline & HR $(95 \%-K I)$ & p-Wert & HR $(95 \%-K I)$ & $\mathrm{p}$-Wert & HR $(95 \%-K I)$ & $\mathrm{p}$-Wert \\
\hline \multicolumn{7}{|l|}{ Ventrikuläre Ektopie: } \\
\hline VES $\geq 313 / 24 \mathrm{~h}$ (Median) & $1,9(0,77-4,69)$ & 0,16 & $2,58(0,95-6,99)$ & 0,06 & $1,96(0,99-3,89)$ & 0,05 \\
\hline $\mathrm{nsVT} \geq 1 / 24 \mathrm{~h}$ & $1,62(0,72-3,66)$ & 0,24 & $2,17(0,87-5,38)$ & 0,1 & $1,91(1,01-3,61)$ & 0,05 \\
\hline \multicolumn{7}{|l|}{$\frac{\text { Herzfrequenzvariabilität: }}{\text { a.) zeitbezogene HRV-Parameter: }}$} \\
\hline SDNNi $<36$ ms (Median) & $1,01(0,45-2,25)$ & 0,98 & $1,09(0,44-2,67)$ & 0,86 & $1,31(0,6-2,87)$ & 0,88 \\
\hline SDNN $<90 \mathrm{~ms}$ (Median) & $1,74(0,76-4)$ & 0,57 & $1,25(0,51-3,08)$ & 0,62 & $1,6(0,84-3,03)$ & 0,15 \\
\hline SNN50Gesamt $<2406$ (Median) & $0,93(0,4-2,16)$ & 0,86 & $1,68(0,68-4,17)$ & 0,26 & $1,18(0,63-2,23)$ & 0,6 \\
\hline RMSSD < 21 ms (Median) & $0,73(0,31-1,69)$ & 0,46 & $1,63(0,66-4,03)$ & 0,29 & $1,02(0,54-1,91)$ & 0,96 \\
\hline \multicolumn{7}{|l|}{ b.) frequenzbezogene HRV-Parameter: } \\
\hline VLF $>898 \mathrm{~ms}^{2}$ (Median) & $0,73(0,33-1,63)$ & 0,45 & $1,06(0,44-2,59)$ & 0,89 & $0,86(0,46-1,6)$ & 0,64 \\
\hline $\mathrm{LF}>226 \mathrm{~ms}^{2}$ (Median) & $1,44(0,64-3,26)$ & 0,38 & $1,41(0,57-3,51)$ & 0,46 & $1,48(0,78-2,8)$ & 0,68 \\
\hline $\mathrm{HF}<112 \mathrm{~ms}^{2}$ (Median) & $0,78(0,34-1,78)$ & 0,55 & $2(0,79-5,05)$ & 0,15 & $1,15(0,61-2,14)$ & 0,67 \\
\hline $\mathrm{LF} / \mathrm{HF}<2,04$ (Median) & $0,98(0,41-2,33)$ & 0,96 & $1,18(0,46-3,05)$ & 0,73 & $0,95(0,49-1,86)$ & 0,89 \\
\hline \multicolumn{7}{|l|}{ Herzfrequenzturbulenz: } \\
\hline $\mathrm{TO}<0 \%$ & $1,06(0,47-2,4)$ & 0,88 & $1,66(0,65-4,26)$ & 0,29 & $1,41(0,74-2,69)$ & 0,3 \\
\hline $\mathrm{TS}<2,5 \mathrm{~ms} / \mathrm{RR}-\mathrm{I}$. & $1,42(0,58-3,48)$ & 0,44 & $1,87(0,69-5,03)$ & 0,22 & $1,91(0,95-3,86)$ & 0,07 \\
\hline \multicolumn{7}{|l|}{ Kategorien der Herzfrequenzturbulenz: } \\
\hline HRT 1 vs. HRT 0 & $1,33(0,42-4,21)$ & 0,63 & $3,36(0,85-13,32)$ & 0,09 & $2,18(0,86-5,35)$ & 0,1 \\
\hline HRT 2 vs. HRT 0 & $1,42(0,47-4,26)$ & 0,53 & $3,03(0,77-12,05)$ & 0,11 & $2,34(1,21-6,76)$ & 0,06 \\
\hline HRT 2 vs. HRT 1 & $1,07(0,43-2,64)$ & 0,89 & $0,91(0,35-2,35)$ & 0,84 & $1,09(0,55-2,16)$ & 0,81 \\
\hline \multicolumn{7}{|l|}{ Dezelerations- und Akzelerationskapazität: } \\
\hline \multicolumn{7}{|l|}{ Kategorien der Dezelerationskapazität: } \\
\hline $\mathrm{DC} \leq 2,5 \mathrm{~ms}$ vs. $\mathrm{DC}>2,5-\leq 4,5 \mathrm{~ms}$ & $1,22(0,5-2,97)$ & 0,66 & $1,95(0,63-6,02)$ & 0,25 & $1,33(0,48-3,72)$ & 0,58 \\
\hline $\mathrm{DC} \leq 2,5 \mathrm{~ms}$ vs. $\mathrm{DC}>4,5 \mathrm{~ms}$ & $1,56(0,44-5,59)$ & 0,49 & $2,51(0,65-9,71)$ & 0,18 & $1,9(0,75-4,83)$ & 0,18 \\
\hline $\mathrm{DC}>2,5-\leq 4,5 \mathrm{~ms}$ vs. $\mathrm{DC}>4,5 \mathrm{~ms}$ & $1,91(0,5-7,27)$ & 0,34 & $1,29(0,28-6,01)$ & 0,75 & $1,43(0,68-3)$ & 0,35 \\
\hline Akzelerationskapazität $>-6,4 \mathrm{~ms}$ (Median) & $1,16(0,52-2,58)$ & 0,72 & $0,76(0,31-1,83)$ & 0,53 & $0,81(0,44-1,51)$ & 0,51 \\
\hline
\end{tabular}

Fett-Kursiv: statistisch signifikante Risikomarker 


\section{Diskussion}

Die Ergebnisse der vorliegenden Arbeit zeigen, dass in einem Studienregister mit heterogenen Patienten mit einem neu implantierten ICD ohne Selektion nach einer kardialen Grunderkrankung keiner der untersuchten LZ-EKG-Risikomarker eine ausreichend starke Korrelation mit den klinischen Endpunkten Gesamtmortalität oder Auftreten des ersten appropiaten ICD-Schock aufwies, um daraus eine sichere Veränderung in der Indikationsstellung zur ICD-Therapie abzuleiten.

Mit der ventrikulären Extrasystolie, dem Auftreten von nsVT und der Kategorisierung der Herzfrequenzturbulenz lagen Risikomarker vor, für die in dieser Arbeit ein statistischer Trend für ein erhöhtes Risiko von appropiaten ICD-Schocks aufgezeigt wurde. Das Auftreten einer nsVT zeigte auch in einem durch die ICD-Indikation erheblich vorselektierten Studienregister unabhängig vom Alter und Geschlecht des Patienten statistisch signifikant ein moderat erhöhtes Risiko für das Erreichen eines unspezifischen Endpunktes aus Gesamtmortalität und Auftreten des ersten appropiaten ICD-Schocks an.

\subsection{Prognostische Relevanz von LZ-EKG-Risikomarkern für Patienten mit einem ICD}

Eine Bewertung der prognostischen Relevanz von LZ-EKG-Risikomarkern in einem Studienregister, das ausschließlich aus Patienten mit implantiertem ICD ohne Selektion nach einer kardialen Vorerkrankung besteht, ist bisher nicht erfolgt. Die bisherigen Arbeiten über die prognostische Relevanz von LZ-EKG-Risikomarkern wurden vor allem an Patienten mit einem akuten Myokardinfarkt oder einer chronischen Herzinsuffizienz im Rahmen einer ischämischen oder nicht-ischämischen Kardiomyopathie durchgeführt (Crawford et al. 1999; Goldberger et al. 2008). Insbesondere für Patienten mit einer dauerhaft hochgradig eingeschränkten LVEF $<35$ \% und damit dem Großteil der Patienten mit einem ICD liegen nur wenige Arbeiten mit zum Teil widersprüchlichen Ergebnissen vor (Goldberger et al. 2008).

Die GESICA-GEMA-Studie (Grupo de Estudio de la Sobrevida en la Insuficienca Cardiaca en Argentina-Grupo de Estudios Multicentricos en Argentina), in der 516 Patienten mit einer chronischen Herzinsuffizienz (39\% durch ICM), einer mittleren LVEF von $19 \%$ und einer Nachbeobachtungszeit von 24 Monaten untersucht wurden, berichtete von einem unabhängigen Zusammenhang zwischen dem Auftreten von $\geq 1 \mathrm{nsVT} / 24 \mathrm{~h}$ und einem erhöhten SCD-Risiko (RR: 2,77; 95 \%-KI 1,78-4,44; p<0,01) sowie von einem erhöhten 
Risiko der Gesamtmortalität (RR: 1,69; 95 \%-KI 1,27-2,24; $<<0,01$; Doval et al. 1996). In der CHF-STAT-Studie (Survival Trial of Antiarrhytmic Therapy in Congestive Heart Failure) über 666 Patienten mit einer chronischen Herzinsuffizienz (71 \% durch ICM), einer mittleren LVEF von $26 \%$ und einer Nachbeobachtungszeit von 45 Monaten konnte keine signifikante Risikoerhöhung durch das Auftreten von $\geq 1 \mathrm{nsVT} / 24 \mathrm{~h}$ für den Endpunkt SCD aufgezeigt werden und für den Endpunkt Gesamtmortalität bestand nur ein Trend ( $p=0,07$; Singh et al. 1998). In der PROMISE-Studie (Prospective Randomized Milrinone Survival Evaluation) über 1.080 Patienten mit einer chronischen Herzinsuffizienz (55\% durch ICM), einer mittleren LVEF von $21 \%$ und einer mittleren Nachbeobachtungszeit von 6 Monaten zeigte in der multivariaten Analyse die Quantifizierung der nsVT sowohl eine schwache Risikoerhöhung für einen SCD (RR: 1,16; $95 \%$-KI 1,09-1,24; $\mathrm{p}<0,01$ ) als auch für die Mortalität durch andere Ursachen (RR: 1,12; 95 \%-KI 1,07-1,17; $<<0,01$ ) auf, jedoch ohne eine spezifische Unterscheidung zwischen diesen Endpunkten zu ermöglichen (Teerlink et al. 2000). Die Quantifizierung der VES zeigte in der PROMISE-Studie keine Risikoerhöhung für einen der Endpunkte an.

Ein Vorteil der LZ-EKG-Parameter der ventrikulären Ektopie gegenüber den Parametern der HRV, der HRT, der DC und der AC besteht darin, dass auch unter Beachtung der hohen Prävalenz (13-27\%) des Vorhofflimmerns bei Patienten mit schweren strukturellen Herzerkrankungen, das Vorliegen eines Sinusrhythmus nicht erforderlich ist (Anter et al. 2009).

Neben den LZ-EKG-Parametern der ventrikulären Ektopie ist auch die prognostische Relevanz der Parameter der HRV für Patienten mit einer stark eingeschränkten LVEF unklar (Goldberger et al. 2008). Die Vielzahl an unterschiedlichen Methoden zur Bestimmung der HRV schränkt die Vergleichbarkeit mit den vorherigen Arbeiten deutlich ein (TASK 1996). Der bisher am häufigsten verwendete HRV-Parameter ist der SDNN-Wert. So wiesen in der prospektiven UK-Heart-Studie (United Kingdom Heart Failure Evaluation and Assessment of Risk Trial) über 433 Patienten mit einer chronischen Herzinsuffizienz (76\% durch ICM), einer mittleren LVEF von $41 \%$ und einer mittleren Nachbeobachtungszeit von 482 Tagen die Patienten mit einem SDNN-Wert $<50 \mathrm{~ms}$ eine wesentlich höhere Mortalitätsrate $(51 \%$ pro Jahr) als die Patienten mit einem SDNN-Wert $>100 \mathrm{~ms}$ (6\% pro Jahr) auf (Nolan et al. 1998). Bei den Überlebenden wurde in dieser Arbeit zwar im Durchschnitt ein signifikant höherer SDNN-Wert gemessen als bei den Patienten, die durch das Fortschreiten ihrer Herzinsuffizienz verstarben $(\mathrm{p}<0,01)$, jedoch bestand kein entsprechender Unterschied im 
Vergleich mit den Patienten, die durch einen SCD verstarben. In einer kleineren Arbeit über 127 Patienten mit einer chronischen Herzinsuffizienz (75\% durch ICM), einer mittleren LVEF von $26 \%$ und einer mittleren Nachbeobachtungszeit von 34 Monaten zeigte ein SDNN-Wert $<65,3 \mathrm{~ms}$ als unabhängiger Risikomarker eine erhöhte Gesamtmortalität an (RR: 3,72; $\mathrm{p}<0,01$ ) und wies für den Endpunkt SCD einen Trend (RR: 2,4; $\mathrm{p}=0,09$ ) auf (Bilchick et al. 2002).

Auch zur Bewertung der prognostischen Relevanz der HRT für Patienten mit einer strukturellen Herzerkrankung und hochgradiger LVEF-Einschränkung stehen nur wenige Arbeiten zur Verfügung. So berichtet eine Arbeit über 358 Patienten, die ebenfalls im Rahmen der UK-Heart-Studie (74 \% mit einer ICM; mittlere LVEF: $41 \%$ ) rekrutiert wurden, nach einer mittleren Nachbeobachtungszeit von fünf Jahren über einen signifikant unabhängigen Zusammenhang (HR: 0,84; 95 \%-KI: 1,09-1,42; p=0,01) zwischen einem niedrigen TS-Wert und dem Erreichen des Endpunktes Mortalität durch eine kardiale Dekompensation (Moore et al. 2006). Für den Endpunkt SCD wies in dieser Studie weder der TS noch der TO eine signifikante Korrelation auf. In der MUSIC-Studie (Muerte Subita en Insuficiencia Cardiaca-Trial) über 607 Patienten mit einer chronischen Herzinsuffizienz (50\% durch ICM) und einer mittleren LVEF von $37 \%$ konnte nach einer mittleren Nachbeobachtungszeit von 44 Monaten sowohl für einen TS-Wert $<2,5 \mathrm{~ms} /$ RR-I. als auch für die Zugehörigkeit zur Kategorie HRT 2 eine signifikante Korrelation mit dem Erreichen der Endpunkte Gesamtmortalität (HR HRT $_{2}$ : 2,52; $\left.95 \%-\mathrm{KI}: 1,56-4,05 ; \mathrm{p}<0,01\right)$ und SCD (HR HRT 2: $_{2,25 ;} 95$ \%-KI: 1,13-4,46; $<<0,01$ ) beobachtet werden (Cygankiewicz et al. 2008).

In einer Subgruppen-Analyse der Daten von 388 Patienten (50\% mit einer ICM; mittlere LVEF von $33 \%$ ) aus der GISSI-HF-Studie (Gruppo Italiano per lo Studio della Sopravvivenza nell'Infarto Miocardico-Heart Failure) zeigte nach einer mittleren Nachbeobachtungszeit von 47 Monaten eine niedrige DC in der multivariaten Analyse lediglich einen schwachen Trend für ein erhöhtes Risiko der Mortalität durch kardiovaskuläre Ursachen (HR 1,07; 95 \%-KI: 0,99-2,25) auf (La Rovere et al. 2012). Es konnte keine Korrelation mit der Mortalität durch einen SCD aufgezeigt werden. Die AC wurde in dieser Arbeit nicht untersucht.

Neben der Selektion nach Indikation zur ICD-Therapie unterscheidet sich die vorliegende Arbeit im Vergleich mit den zitierten Studien zum Teil deutlich in der Größe des Studienregisters, der Dauer der Nachbeobachtungszeit, dem Anteil der Patienten mit einer ICM, der Höhe der mittleren LVEF und in unterschiedlichen Grenzwerten der LZ-EKG- 
Risikomarker. Insbesondere die sich ab einer LVEF $<40 \%$ exponentiell erhöhende Verschlechterung des Gesamtüberlebens stellt je nach Studie eine zunehmende Einschränkung in der Vergleichbarkeit mit der vorliegenden Arbeit dar (Buxton et al. 2002; Buxton 2005). In den zitierten älteren Vergleichsstudien wie z. B. der CHF-STAT-Studie oder der GESICA-GEMA-Studie wurden keine Patienten mit einem ICD eingeschlossen (Doval et al. 1996; Singh et al. 1998). Auch in der aktuelleren GISSI-HF-Studie ist mit 7,1\% der Anteil von ICD-Trägern vergleichsweise gering (La Rovere et al. 2012). Ein Beispiel für weitere, bei der Beschreibung der zitierten Studien nicht dargestellte, aber möglicherweise dennoch ergebnisrelevante Einflussgrößen ist die aus heutiger Sicht weniger fortschrittliche medikamentöse Therapie in älteren Studien. So nahmen z. B. in der Arbeit von Bilchick (Bilchick et al. 2002), in der ein niedriger SDNN-Wert im Gegensatz zur vorliegenden Arbeit einen unabhängigen Risikomarker für die Gesamtmortalität darstellte, nur $6 \%$ der Patienten einen Betablocker ein.

Wie dargestellt, bilden die zitierten Arbeiten nicht alle Indikationen zur ICD-Implantation ab. Da klinische Arbeiten über Patienten mit implantierten ICDs jedoch noch fehlen, ist nur ein Vergleich mit diesen Arbeiten möglich. Fasst man die Ergebnisse zusammen, so hat keiner der LZ-EKG-Risikomarker unter Beachtung der geringen und zum Teil widersprüchlichen Studienlage bisher eine so starke Korrelation mit einem der Endpunkte aufgezeigt, um durch die supportive Bestimmung eines der LZ-EKG-Risikomarker eine Änderung der Indikationsstellung zur ICD-Implantation rechtfertigen zu können. Auch die Ergebnisse der vorliegenden Arbeit deuten an, dass für keinen der LZ-EKG-Risikomarker eine ausreichend starke Assoziation vorliegt. 


\subsection{Stärken und Schwächen der vorliegenden Arbeit}

Durch die Selektion nach Indikation zur ICD-Implantation und nicht nach einer bestimmten kardialen Grunderkrankung besitzt das Studienregister in der vorliegenden Arbeit eine hohe Repräsentativität für den klinischen Alltag mit Patienten, die ein diagnostiziertes hohes Risiko für maligne Rhythmusstörungen haben. Eine Bewertung der prognostischen Relevanz von LZ-EKG-Risikomarkern in einem Studienregister, das ausschließlich aus Patienten mit implantiertem ICD ohne Selektion nach einer kardialen Vorerkrankung besteht, ist bisher nicht erfolgt.

Der in dieser Arbeit verwendete Endpunkt des ersten appropiaten ICD-Schocks spiegelt nicht vollständig wider, welche Patienten von der Implantation eines ICDs profitieren, da Patienten, deren Rhythmusstörung bereits durch eine tachykarde Überstimulation des ICDs therapiert wurden, nicht berücksichtig werden. Da viele als SCD klassifizierte Todesfälle jedoch nicht durch eine ventrikuläre Arrhythmie verursacht sind und umgekehrt viele durch eine ventrikuläre Arrhythmie bedingte Todesfälle nicht als SCD klassifiziert werden, spiegelt dieser Endpunkt vermutlich dennoch das Risiko für eine lebensbedrohliche ventrikuläre Arrhythmie besser wider als die Endpunkte Mortalität kardialer Genese oder SCD, welche in früheren Studien verwandt wurden (Epstein et al. 1996; Pratt et al. 1996).

Die geringe Größe des Studienregisters schränkt die Aussagekraft dieser Arbeit deutlich ein. Es besteht die Gefahr, eine bestehende Korrelation bei einer niedrigeren Effektstärke fälschlicherweise nicht aufgedeckt $\mathrm{zu}$ haben (Fehler 2. Art). Die hohen Breiten der Konfidenz-Intervalle verdeutlichen, dass die Größe der in dieser Arbeit untersuchten Stichproben nur eine ungenaue Schätzung der Effektgrößen ermöglicht. Insbesondere für die LZ-EKG-Parameter, die in dieser Arbeit knapp die Signifikanz-Kriterien verfehlt haben, könnte somit in größeren Studien eine signifikante Korrelation nachgewiesen werden. Die geringe Anzahl der klinischen Ereignisse verhinderte den Einschluss mehrerer wichtiger KoVariablen (z. B. kardiale Grunderkrankung; Höhe der LVEF etc.) in die multivariate Analyse. 


\subsection{Schlussfolgerung}

Für eine genauere Bestimmung des prädiktiven Wertes der untersuchten LZ-EKGRisikomarker sind weitere Arbeiten mit größeren Studienregistern von ICD-Trägern erforderlich. Mit der ventrikulären Extrasystolie, dem Vorhandensein nicht-anhaltender ventrikulärer Tachykardien und der Kategorisierung nach Risikogruppen der Herzfrequenzturbulenz liegen LZ-EKG-Risikomarker vor, für die wahrscheinlich in einem größeren Studienregister eine signifikante Assoziation mit dem Auftreten eines appropiaten ICD-Schocks aufgezeigt werden könnte. Die in der vorliegenden Arbeit bestimmten Effektstärken deuten jedoch für keinen dieser LZ-EKG-Risikomarker eine ausreichend starke Risikoerhöhung an, um durch die supportive Bestimmung eines der LZ-EKG-Risikomarker eine deutliche Verbesserung in der therapeutischen Effektivität der ICD-Therapie zu erreichen.

In Zukunft wird deshalb zu prüfen sein, ob es möglicherweise auch mit Hilfe der Kombination verschiedener weiterer nicht-invasiver Verfahren (QRS-Dauer-Bestimmung, TWellen-Alternans-Testung etc.) gelingen kann, die Auswahl der Patienten, die von der Implantation eines ICDs profitieren, weiter zu optimieren. 


\section{Zusammenfassung}

Der plötzliche Herztod durch das Auftreten anhaltender ventrikulärer Arrhythmien ist die häufigste Todesursache in den westlichen Industrienationen. Mit der Implantation eines implantierbaren Kardioverter-Defibrillators steht eine Therapie zur Verfügung, die hocheffektiv lebensbedrohliche Arrhythmien beenden kann. Die Verfahren zur Bestimmung des individuellen Risikos für das Auftreten lebensbedrohlicher Arrhythmien sind verbesserungswürdig, da aktuell einigen Patienten ein ICD implantiert wird, die niemals von diesem Gerät profitieren.

Diese monozentrische Beobachtungsstudie überprüfte an 150 Patienten, denen nach aktueller Indikation ein ICD implantiert wurde, die prognostische Wertigkeit von gängigen LZ-EKGRisikomarkern wie der ventrikulären Ektopie, der Herzfrequenzvariabilität, der Herzfrequenzturbulenz, der Dezelerations- und der Akzelerationskapazität auf das klinische Auskommen. Eine Selektion der Patienten nach einer bestimmten kardialen Grunderkrankung wurde nicht vorgenommen.

Die mittlere Nachbeobachtungszeit betrug $28 \pm 12$ Monate. In dieser Zeit verstarben 25 Patienten und bei 20 Patienten löste der ICD einen appropiaten Schock aus. Keiner der untersuchten LZ-EKG-Risikomarker zeigte unabhängig vom Alter und Geschlecht des Patienten statistisch signifikant ein erhöhtes Risiko für das Erreichen der Endpunkte Gesamtmortalität oder Auftreten eines appropiaten ICD-Schocks an. Für die LZ-EKGRisikomarker ventrikuläre Extrasystolie, nsVT und die Zugehörigkeit zur Kategorisierung HRT 1 konnte unabhängig vom Alter und Geschlecht des Patienten ein statistischer Trend für ein erhöhtes Risiko von appropiaten ICD-Schocks aufgezeigt werden. Lediglich der Risikomarker nsVT zeigte in der multivariaten Analyse statistisch signifikant ein erhöhtes Risiko für das Erreichen eines kombinierten Endpunktes aus Gesamtmortalität und Auftreten eines appropiaten ICD-Schocks an (HR: 1,91 95 \%-KI: 1,01-3,61, p-Wert: 0,05).

Die Größe des untersuchten Studienregisters lässt nur eine ungenaue Berechnung des prädiktiven Wertes der LZ-EKG-Risikomarker zu. Eine abschließende Bewertung der prognostischen Relevanz der bestimmten LZ-EKG-Risikomarker für Patienten mit einem implantierten ICD muss in größeren klinischen Studien erfolgen. 


\section{LITERATURVERZEICHNIS}

Akselrod et al. 1981 = Akselrod S, Gordon D, Ubel FA, Shannon DC, Barger AC, Cohen RJ (1981): Power spectrum analysis of heart rate fluctuation: a quantitative probe of beat to beat cardiovascular control. Science $\underline{213}, 220-222$

Anter et al. 2009 = Anter E, Jessup M, David J, Callans DJ (2009): Atrial Fibrillation and Heart Failure: Treatment Considerations for a Dual Epidemic. Circulation 119, 2516-2525

AVID-Investigators $1997=$ The Antiarrhythmics versus Implantable Defibrillators (AVID) Investigators (1997): A comparison of antiarrhythmic-drug therapy with implantable defibrillators in patients resuscitated from near-fatal ventricular arrhythmias. N Engl J Med $\underline{337}, 1576-1584$

Bailey et al. 2001 = Bailey JJ, Berson AS, Handelsman H, Hodges M (2001): Utility of current risk stratification tests for predicting major arrhythmic events after myocardial infarction. J Am Coll Cardiol $\underline{38}, 1902-1911$

Baldasseroni et al. 2002 = Baldasseroni S, Opasich C, Gorini M, Lucci D, Marchionni N, Marini M, Campana C, Perini G, Deorsola A, Masotti G et al. (2002): Left bundle-branch block is associated with increased 1-year sudden and total mortality rate in 5517 outpatients with congestive heart failure: a report from the Italian network on congestive heart failure. Am Heart J 143, 398-405

Bardy et al. 2005 = Bardy GH, Lee KL, Mark DB, Poole JE, Packer DL, Boineau R, Domanski M, Troutman C, Anderson J, Johnson G et al. (2005): Sudden Cardiac Death in Heart Failure Trial (SCD-HeFT) Investigators: Amiodarone or an implantable cardioverterdefibrillator for congestive heart failure. N Engl J Med 352, 225-237

Barthel et al. 2003 = Barthel P, Schneider R, Bauer A, Ulm K, Schmitt C. Schömig A, Schmidt G (2003): Risk stratification after acute myocardial infarction by heart rate turbulence. Circulation $\underline{108}, 1221-1226$

Bauer et al. 2006a = Bauer A, Kantelhardt JW, Barthel P, Schneider R, Mäkikallio T, Ulm K, Hnatkova K, Schömig A, Huikuri H, Bunde A et al. (2006): Deceleration capacity of heart rate as a predictor of mortality after myocardial infarction: cohort study. Lancet $\underline{367}, 1674-$ 1681 
Bauer et al. 2006b = Bauer A, Kantelhardt JW, Bunde A, Barthel P, Schneider R, Malik M, Schmidt G (2006): Phase-rectified signal averaging detects quasi-periodicities in nonstationary data. Physica A $\underline{364}, 423-434$

Bauer et al. 2008 = Bauer A, Malik M, Schmidt G, Barthel P, Bonnemeier H, Cygankiewicz I., Guzik P, Lombardi F, Müller A, Oto A, et al. (2008): Heart Rate Turbulence: Standards of Measurement, Physiological Interpretation and Clinical Use. J Am Coll Cardiol 52, 13531365

Bayès de Luna et al. 1989 = Bayès de Luna A, Courmel P, Leclercq JF (1989): Ambulatory sudden cardiac death: Mechanisms of production of fatal arrhythmia on the basis of data from 157 cases. Am Heart J 117, 151-159

Bigger et al. 1984 = Bigger JT Jr, Fleiss JL, Kleiger R (1984): The relationships among ventricular arrhythmias, left ventricular dysfunction, and mortality in the 2 years after myocardial infarction. Circulation $\underline{69}, 250-258$

Bigger et al. 1992a = Bigger JT, Fleiss JL, Steinman RC, Rolnitzky LM, Kleiger RE, Rottman JN (1992): Frequency domain measures of heart period variability and mortality after myocardial infarction. Circulation $\underline{85}, 164-171$

Bigger et al. 1992b = Bigger JT Jr, Fleiss JL, Steinman RC, Rolnitzky LM, Kleiger RE, Rottman JN (1992): Correlations among time and frequency domain measures of heart period variability two weeks after acute myocardial infarction. Am J Cardiol 69, 889-891

Bilchick et al. 2002 = Bilchick KC, Fetics B, Djoukeng R, Fisher SG, Fletcher RD, Singh SN, Nevo E, Berger RD (2002): Prognostic value of heart rate variability in chronic congestive heart failure (Veterans affairs survival trial of antiarrhythmic therapy in congestive heart failure). Am J Cardiol 90, 24-28

Bloomfield et al. $2006=$ Bloomfield DM, Bigger JT, Steinman RC, Namerow PB, Parides MK, Curtis AB, Kaufman ES, Davidenko JM, Shinn TS, Fontaine JM (2006): Microvolt Twave alternans and the risk of death or sustained ventricular arrhythmias in patients with left ventricular dysfunction. J Am Coll Cardiol 47:456-463

Buxton 2005 = Buxton AE (2005): Should everyone with an ejection fraction less than or equal to $30 \%$ receive an implantable cardioverter-defibrillator? Not everyone with an ejection 
fraction $<$ or $=30 \%$ should receive an implantable cardioverter-defibrillator. Circulation $\underline{111}$, 2537-2549

Buxton 2009 = Buxton AE (2009): Risk stratification for sudden death in patients with coronary artery diease. Heart Rhythm $\underline{6}, 836-847$

Buxton et al. 1999 = Buxton AE, Lee KL, Fisher JD, Josephson ME, Prystowsky EN, Hafley G (1999): A randomized study of the prevention of sudden death in patients with coronary artery disease. Multicenter Unsustained Tachycardia Trial Investigators. N Engl J Med $\underline{341}$, 1882-1890

Buxton et al. 2000 = Buxton AE, Lee KL, DiCarlo L, Gold MR, Greer GS, Prystowsky EN, O'Toole MF, Tang A, Fisher JD, Coromilas J et al. (2000): Electrophysiologic testing to identify patients with coronary artery disease who are at risk for sudden death. N Engl J Med $\underline{342}, 1937-1945$

Buxton et al. 2002 = Buxton AE, Lee KL, Hafley GE, Wyse DG, Fisher JD, Lehmann MH, Pires LA, Gold MR, Packer DL, Josephson ME et al. (2002): Relation of ejection fraction and inducible ventricular tachycardia to mode of death in patients with coronary artery disease: an analysis of patients enrolled in the multicenter unsustained tachycardia trial. Circulation $\underline{106}$, $2466-2472$

Connolly et al. 2000a = Connolly SJ, Gent M, Roberts RS, Dorian P, Roy D, Sheldon RS, Mitchell LB, Green MS, Klein GJ, O'Brien B (2000a): Canadian implantable defibrillator study (CIDS): a randomized trial of the implantable cardioverter defibrillator against amiodarone. Circulation 101, 1297-1302

Connolly et al. 2000b = Connolly SJ, Hallstrom AP, Cappato R, Schron EB, Kuck KH, Zipes OP, Greene Hl, Boczor S, Oomanski M, Fallmann O et al. (2000b): Meta-analysis of the implantable cardioverter defibrillator secondary prevention trials. AVID, CASH and CIDS studies. Antiarrhythmics vs Implantable Defibrillator study. Cardiac Arrest Study Hamburg. Canadian Implantable Defibrillator Study. Eur Heart J 21, 2071-2078

Costantini et al. $2009=$ Costantini O, Hohnloser SH, Kirk MM, Lerman BB, Baker JH, Sethuraman B, Dettmer MM, Rosenbaum DS, for the ABCD Trial Investigators (2009): The ABCD (Alternans Before Cardioverter Defibrillator) Trial Strategies Using T-Wave Alternans to Improve Efficiency of Sudden Cardiac Death Prevention. J Am Coll Cardiol $\underline{53}$, 471-479 
Crawford et al. 1999 = Crawford MH, Bernstein SJ, Deedwania PC, DiMarco JP, Ferrick KJ, Garson A Jr, Green LA, Greene HL, Silka MJ, Stone PH et al. (1999): ACC/AHA Guidelines for Ambulatory Electrocardiography. A report of the American College of Cardiology/ American Heart Association Task Force on Practice Guidelines (Committee to Revise the Guidelines for Ambulatory Electrocardiography). Developed in collaboration with the North American Society for Pacing and Electrophysiology. J Am Coll Cardiol 34, 912-948

Cygankiewicz et al. 2008 = Cygankiewicz I, Zareba W, Vazquez R, Gonzalez-Juanately JR, Valdes M., Almendral J, Cinca J, Caminal P, de Luna AB, Muerte Subita en Insuficienca Cardiaca Investigators (2008): Heart rate turbulence predicts all-cause mortality and sudden death in congestive heart failure patients. Heart Rhythm $\underline{5}, 1095-1102$

Daubert et al. 2008 = Daubert JP, Zareba W, Cannom OS, McNitt S, Rosero SZ, Wang P, Schuger C, Steinberg JS, Higgins SL, Wilber OJ et al. (2008): Inappropriate implantable cardioverter-defibrillator shocks in MADIT II: frequency, mechanisms, predictors, and survival impact. J Am Coll Cardiol 51, 1357-1365

Denes et al. 1991 = Denes P, Gillis AM, Pawitan Y, Kammerling JM, Wilhelmsen L, Salerno DM (1991): Prevalence, characteristics and significance of ventricular premature complexes and ventricular tachycardia detected by 24 -hour continuous electrocardiographic recording in the Cardiac Arrhythmia Suppression Trial. CAST Investigators. Am J Cardiol 68, 887-896.

DiMarco 2003 = DiMarco JP (2003): Implantable Cardioverter-Defibrillators. N Eng J Med $\underline{349}, 1836-1847$

Doval et al. 1996 = Doval HC, Nul DR, Grancelli HO, Varini SD, Soifer S, Corrado G, Dubner S, Scapin O, Perrone SV (1996): Nonsustained ventricular tachycardia in severe heart failure: independent marker of increased mortality due to sudden death: GESICA-GEMA Investigators. Circulation 94, 3198-3203

El-Sherif et al. 1990 = El-Sherif N, Gough WB, Restivo M, Craelius W, Henkin R, Caref EB.(1990): Electrophysiological basis of ventricular late potentials. Pacing Clin Electrophysiol $\underline{13}, 2140-2147$

Epstein et al. 1996 = Epstein AE, Carlson MD, Fogoros RN, Higgins SL, Venditti FJ Jr (1996): Classification of death in antiarrhythmia trials. J Am Coll Cardiol 27, 433-442 
Epstein et al. $2008=$ Epstein AE, DiMarco JP, Ellenbogen KA, Estes M III, Freedman RA, Gettes LS, Gillinov AM, Gregoratos G, Hammill SC, Hayes DL et al. (2008): ACC/AHA/HRS 2008 Guidelines for Device-Based Therapy of Cardiac Rhythm Abnormalities: Executive Summary: A Report of the American College of Cardiology/American Heart Association Task Force on Practice Guidelines. Circulation 117, $2820-2840$

Ewing et al. 1984 = Ewing DJ, Neilson JM, Travis P (1984): New method for assessing cardiac parasympathetic activity using 24 hour electrocardiograms. Br Heart J 52, 396-402

Exner et al. 2007 = Exner DV, Kavanagh KM, Slawnych MP, Mitchell LB, Ramadan D, Aggarwal SG, Noullett C, Van Schaik A, Mitchell RT, Shibata MA et al. (2007): Noninvasive risk assessment early after a myocardial infarction the REFINE study. J Am Coll Cardiol 50, 2275-2284

Farrell et al. $1991=$ Farrell TG, Bashir Y, Cripps T, Malik M, Poloniecki J, Bennett ED, Ward DE, Camm AJ. (1991): Risk stratification for arrhythmic events in postinfarction patients based on heart rate variability, ambulatory electrocardiographic variables and the signal- averaged electrocardiogram. J Am Coll Cardiol 18, 687-697

Gardner et al. $1985=$ Gardner PI, Ursell PC, Fenoglio JJ Jr, Wit AL (1985): Electrophysiologic and anatomic basis for fractionated electrograms recorded from healed myocardial infarcts. Circulation $\underline{72}$, 596-611

Gehi et al. 2005 = Gehi AK, Stein RH, Metz LD, Gomes JA. (2005): Microvolt T-wave alternans for the risk stratification of ventricular tachyarrhythmic events: a meta-analysis. $\mathrm{J}$ Am Coll Cardiol $\underline{46}, 75-82$

Ghuran et al. 2002 = Ghuran A, Reid F, La Rovere MT, Schmidt G, Bigger JT, Camm AJ, Schwartz PJ, Malik M (2002): Heart rate turbulence-based predictors of fatal and nonfatal cardiac arrest (The Autonomic Tone and Reflexes After Myocardial Infarction substudy). Am J Cardiol $\underline{89}$, 184-190

Goldberger et al. 2008 = Goldberger JJ, Cain ME, Hohnloser SH, Kadish AH, Knight BP. Lauer MS, Maron BJ, Page RL, Passman RS, Siscovick D et al. (2008): American Heart Association/American College of Cardiology Foundation/Heart Rhythm Society Scientific Statement on Noninvasive Risk Stratification Techniques for Identifying Patients at Risk for 
Sudden Cardiac Death: A Scientific Statement From the American Heart Association Council on Clinical Cardiology Committee on Electrocardiography and Arrhythmias and Council on Epidemiology and Prevention. Circulation $\underline{118}, 1497-1518$

Hallstrom et al. 2005 = Hallstrom AP, Stein PK, Schneider R, Hodges M, Schmidt G, Ulm K, for the CAST Investigators (2005): Characteristics of heart beat intervals and prediction of death. Int J Cardiol 100, 37-45

Heller et al. 1998 = Heller SS, Ormont MA, Lidagoster L, Sciacca RR, Steinberg S. (1998): Psychosocial outcome after ICD implantation: a current perspective. Pacing Clin Electrophysiol 21, 1207-1215

Huikuri et al. 2001= Huikuri HV, Castellanos A, Myerburg RJ (2001): Sudden Death Due to Cardiac Arrhythmias. N Engl J Med 345, 1473-1482

Jalife et al. 1983 = Jalife J, Slenter VAJ, Salata JJ, Michaels DC.(1983): Dynamic vagal control of pacemaker activity in the mammalian sinoatrial node. Circ Res $\underline{52}, 642-656$.

Jodko et al. 2009 = Jodko Ł, Kornacewicz-Jach Z, Kaźmierczak J, Rzeuski R, Zielonka J, Kaliszczak R, Safranow K. (2009): Inappropriate cardioverter-defibrillator discharge continues to be a major problem in clinical practice.. Cardiol J $\underline{16}, 432-439$

Kadish et al. 2004 = Kadish A, Dyer A, Daubert JP, Quigg R, Estes M, Anderson KP, Calkins H, Hoch D, Goldberger J, Shalaby A et al. (2004): Prophylactic defibrillator implantation in patients with nonischemic dilated cardiomyopathy. N Engl J Med 350, 2151-2158

Kamphuis et al. 2003 = Kamphuis HC, de Leeuw JR, Derksen R, Hauer RN, Winnubst JA (2003): Implantable cardioverter defibrillator recipients: quality of life in recipients with and without ICD shock delivery: a prospective study. Europace $\underline{5}$, 381-389

Kuck et al. $2000=$ Kuck KH, Cappato R, Siebels J, Ruppel R (2000): Randomized comparison of antiarrhythmic drug therapy with implantable defibrillators in patients resuscitated from cardiac arrest: the Cardiac Arrest Study Hamburg (CASH). Circulation 102, $748-754$

Kurl et al. 2012 = Kurl S, Mäkikallio TH, Rautahrju P, Kiviniemi V, Laukkanen JA (2012): Duration of QRS Complex in Resting Electrocardiogram is a Predictor of Sudden Cardiac Death in MenClinical Perspective. Circulation 125, 2588-2594 
La Rovere et al. 1998 = La Rovere MT, Bigger JT Jr, Marcus FI, Mortara A, Schwartz PJ. (1998): Baroreflex sensitivity and heart-rate variability in prediction of total cardiac mortality after myocardial infarction. ATRAMI (Autonomic Tone and Reflexes After Myocardial Infarction) Investigators. Lancet $\underline{351}, 478-484$

La Rovere et al. 2001 = La Rovere MT, Pinna GD, Hohnloser SH, Marcus FI, Mortara A, Nohara R, Bigger JT Jr, Camm AJ, Schwartz PJ (2001): Baroreflex sensitivity and heart rate variability in the identification of patients at risk for life-threatening arrhythmias: implications for clinical trials. Circulation $\underline{103}, 2072-2077$

La Rovere et al. 2012 = La Rovere MT, Pinna GD, Maestri R, Barlera S, Bernardinangeli M, Veniani M, Nicolosi GL, Marchioli R, Tavazzi L; GISSI-HF Investigators (2012): Autonomic markers and cardiovascular and arrhythmic events in heart failure patients: still a place in prognostication? Data from the GISSI-HF trial. Eur J Heart Fail 14

Maggioni et al. 1993 = Maggioni AP, Zuanetti G, Franzosi MG, Rovelli F, Santoro E, Staszewsky L, Tavazzi L, Tognoni G (1993): Prevalence and prognostic significance of ventricular arrhythmias after acute myocardial infarction in the fibrinolytic era. GISSI-2 results. Circulation $\underline{87}, 312-322$

Makikallio et al. (2005): Makikallio TH, Barthel P, Schneider R, Bauer A, Tapanainen JM, Tulppo MP, Schmidt G, Huikuri HV (2005): Prediction of sudden cardiac death after acute myocardial infarction: role of Holter monitoring in the modern treatment era. Eur. Heart $\mathbf{J} \underline{26}$, 762-769

Markewitz $2014=$ Markewitz A. (2014): Jahresbericht 2012 des Deutschen Herzschrittmacher- und Defibrillatorregisters. Hrsg.: Deutsches Herzschrittmacher und Defibrillatorregister, Fachgruppe Herzschrittmacher und Institut für angewandte Qualitätsforschung im Gesundheitswesen GmbH (AQUA), Göttingen

Mirowski et al. $1980=$ Mirowski M, Reid PR, Mower MM, Watkins L, Gott VL, Schauble JF, langer A, Heilman MS, Kolenik SA, Fischell RE, et al. (1980): Termination of malignant ventricular arrhythmias with an implanted automatic defibrillator in human beings. $\mathrm{N}$ Engl $\mathrm{J}$ Med $\underline{303}, 322-324$ 
Moore et al. 2006 = Moore RK, Groves DG, Barlow PE, Fox KA, Shah A, Nolan J, Kearney MT (2006): Heart rate turbulence and death due to cardiac decompensation in patients with chronic heart failure. Eur J Heart Fail $\underline{8}, 585-590$

Moss et al. 1996 = Moss AJ, Hall WJ, Cannom DS, Daubert JP, Higgins SL, Klein H, Levine JH, Saksena S, Waldo AL, Wilber D et al. (1996): Improved survival with an implanted defibrillator in patients with coronary disease at high risk for ventricular arrhythmia. N Engl J Med 335, 1933-1940

Moss et al. 2002 = Moss AJ, Zareba W, Hall WJ, Klein H, Wilber DJ, Cannom DS, Daubert JP, Higgins SL, Brown MW, Andrews ML (2002): Prophylactic implantation of a defibrillator in patients with myocardial infarction and reduced ejection fraction. N Engl J Med $\underline{346}, 877-$ 883

Moss et al. 2012 = Moss AJ, Schuger C, Beck CA, Brown MW, Cannom DS, Daubert JP, Estes NA 3rd, Greenberg H, Hall WJ, Huang DT et al. (2012): Reduction in inappropriate therapy and mortality through ICD programming. N Engl J Med 367, 2275-2283

Myerburg et al. 1993 = Myerburg RJ, Kessler KM, Castellanos A (1993): Sudden cardiac death: epidemiology, transient risk, and intervention assessment. Ann Intern Med 119, $1187-$ 1197

Myerburg et al. 1997 = Myerburg RJ, Interian A Jr, Mitrani RM, Kessler KM, Castellanos A (1997): Frequency of sudden cardiac death and profiles of risk. Am J Cardiol 무, 10F-19F.

Nolan et al. 1998 = Nolan J, Batin PD, Andrews R, Lindsay S, Brooksby P, Mullen M, Baig W, Flapan A, Cowley A, Prescott R, Neilson J, Fox K (1998): Prospective study of heart rate variability and mortality in chronic heart failure: Results of the United Kingdom heart failure evaluation and assessment of risk trial (UK-heart). Circulation $\underline{98}, 1510-1516$

Oppenheim und Schafer 1975 = Oppenheim AV und Schafer RW (1975): Digital Signal Processing 1. Auflage. Hrsg.: Prentice-Hall, Englewood Cliffs (NJ)

Padmanabhan et al. 2003 = Padmanabhan S, Silvet H, Amin J, Pai RG. (2003): Prognostic value of QT interval and QT dispersion in patients with left ventricular systolic dysfunction: results from a cohort of 2265 patients with an ejection fraction $\leq 40 \%$. Am Heart J 145, 132138 
Poole et al. 2008 = Poole JE, Johnson GW, Hellkamp AS, Anderson J, Callans DJ, Raitt MH, Reddy RK, Marchlinski FE, Yee R, Guarnieri T et al. (2008): Prognostic importance of defibrillator shocks in patients with heart failure. N Engl J Med 359, 1009-1017

Pratt et al. 1996 = Pratt CM, Greenway PS, Schoenfeld MH, Hibben ML, Reiffel JA (1996): Exploration of the Precision of Classifying Sudden Cardiac Death Implications for the Interpretation of Clinical Trials. Circulation $\underline{93}, 519-524$

Rosenbaum et al. 1994 = Rosenbaum DS, Jackson LE, Smith JM, Garan H, Ruskin JN, Cohen RJ. (1994): Electrical alternans and vulnerability to ventricular arrhythmias. N Engl J Med $\underline{330}, 235-241$

Schmidt et al. 1999 = Schmidt G, Malik M, Barthel P, Schneider R, Ulm K, Rolnitzky L, Camm AJ, Bigger JT, Schömig A (1999): Heart-rate turbulence after ventricular premature beats as a predictor of mortality after acute myocardial infarction. Lancet $\underline{353}, 1390-1396$

Schwartz et al. 1992 = Schwartz PJ, La Rovere MT, Vanoli E (1992): Autonomic nervous system and sudden cardiac death. Experimental basis and clinical observations for postmyocardial infarction risk stratification. Circulation $\underline{85}$, 177-191

Schneider 2006 = Schneider H. (2006): Implantate-Katalog Herzrhythmussteuerung 2006. Hrsg.: Medizinischer Dienst des Spitzenverbandes Bund der Krankenkassen e.v., Essen

Scirica et al. 2010 = Scirica BM, Braunwald E, Belardinelli L, Hedgepeth CM, Spinar J, Wang W, Qin J, Karwatowska-Prokopczuk E, Verheugt FW, Morrow DA (2010): Relationship between nonsustained ventricular tachycardia after non-ST-elevation acute coronary syndrome and sudden cardiac death: observations from the metabolic efficiency with ranolazine for less ischemia in non-ST-elevation acute coronary syndrome-thrombolysis in myocardial infarction 36 (MERLIN-TIMI 36) randomized controlled trial. Circulation $\underline{122}$, $455-462$

Singh et al. 1998 = Singh SN, Fisher SG, Carson PE, Fletcher RD (1998): Prevalence and significance of nonsustained ventricular tachycardia in patients with premature ventricular contractions and heart failure treated with vasodilator therapy. J Am Coll Cardiol $\underline{32}$, 942-947

Smith et al. 1995 = Smith ML, Ellenbogen KA, Eckberg DL (1995): Baseline arterial pressure affects sympathoexcitatory responses to ventricular premature beats. Am J Physiol $\underline{269}$, H153-H159 
TASK 1996 = Task Force of The European Society of Cardiology and The North American Society of Pacing and Electrophysiology (1996): Heart rate variability: Standards of measurement, physiological interpretation and clinical use. Eur Heart J 17, 354-381

Teerlink et al. $2000=$ Teerlink JR, Jalaluddin M, Anderson S, Kukin M, Eichhorn E, Francis G, Packer M, Massie B. (2000): Ambulatory ventricular arrhythmias in patients with heart failure do not specifically predict an increased risk of sudden death. PROMISE (Prospective Randomized Milrinone Survival Evaluation) Investigators. Circulation 101, 40-46

Van Rees et al. 2011 = van Rees JB, Borleffs CJW, de Bie MK, Stijnen T, van Erven L, Bax JJ, Schalij MJ (2011): Inappropriate Implantable Cardioverter-Defibrillator Shocks Incidence, Predictors and Impact on Mortality J Am Coll Cardiol 57, 556-562

Vanoli et al. 1991 = Vanoli E, De Ferrari GM, Stramba-Badiale M, Hull SS Jr, Foreman RD, Schwartz PJ (1991): Vagal stimulation and prevention of sudden death in conscious dogs with a healed myocardial infarction. Cir Res $\underline{68}, 1471-1481$

Vollmann et al. 2005 = Vollmann D, Luthje L, Vonhof S, Unterberg C (2005): lnappropriate therapy and fatal proarrhythmia by an implantable cardioverter-defibrillator. Heart Rhythm $\underline{3}$, 307-309

Wathen et al. 2001 = Wathen MS, Sweeney MO, De Groot PJ, Stark AJ, Koehler JL, Chisner MB, Machado C, Adkisson WO, PainFREE Investigators (2001): Shock reduction using antitachycardia pacing for spontaneous rapid ventricular tachycardia in patients with coronary artery disease. Circulation $\underline{104}, 796-801$

Welch et al. 1989 = Welch WJ, Smith ML, Rea RF, Bauernfein RA, Eckberg DL (1989): Enhancement of sympathetic nerve activity by single premature ventricular beats in humans. $\mathrm{J}$ Am Coll Cardiol $\underline{13}, 69-75$

Zipes und Welles 1998 = Zipes DP und Wellens HJJ (1998): Sudden cardiac death. Circulation $\underline{98}, 2334-2351$ 


\section{Danksagung}

An erster Stelle bedanke ich mich bei Herrn Professor Dr. med. Markus Zabel für die freundliche Überlassung des Themas.

Besonders herzlichen Dank möchte ich auch an Herrn Dr. med. Joachim Seegers für seine Betreuung bei der Erstellung dieser Arbeit richten.

Den Mitarbeitern der Abteilung Medizinische Statistik danke ich für ihre Hilfe bei der statistischen Analyse der Ergebnisse. 


\section{Lebenslauf}

Als zweites Kind meiner Eltern Annette Wessels geb. Többen und Ansgar Bernhard Wessels wurde ich am 14.02.1984 in Haselünne geboren. Von 1990 bis 1994 besuchte ich die Grundschule in meinem Heimatort Hemsen und daran anschließend zwei Jahre lang die Orientierungsstufe der Kardinal-von Galen-Schule in Meppen. Von 1996 bis 2003 besuchte ich das Windthorst-Gymnasium in Meppen. Nach dem Abitur war ich im Rahmen meines Zivildienstes Rettungshelfer auf der Rettungswache des DRK in Meppen. Im Verlauf des Zivildienstes schloss ich eine Ausbildung zum Rettungssanitäter ab. Zum Wintersemester 2004 begann ich das Studium der Humanmedizin an der Georg-August-Universität in Göttingen. Nach dem 2. Staatsexamen im Juni 2011 nahm ich eine Tätigkeit als Assistenzarzt in der Abteilung für Allgemeinmedizin der Universitätsmedizin Greifswald unter der Leitung von Herrn Professor Dr. med. Jean-Francois Chenot auf. Seit April 2014 arbeite ich als Assistenzarzt im Diabetes Zentrum Quakenbrück unter Leitung von Herrn Professor Dr. med. Stephan Matthaei. 